\title{
Origin of the gases released from the Acqua Passante and Ermeta wells (Mt. Amiata, central Italy) and possible environmental implications for their closure
}

\author{
Barbara Nisi ${ }^{1, \star}$, Orlando Vaselli ${ }^{2,3}$, Franco Tassi ${ }^{2,3}$, Javier de Elio ${ }^{4}$, Marcelo Ortega ${ }^{4}$, \\ Juan Caballero ${ }^{4}$, Daniele Rappuoli ${ }^{5}$, Luis Felipe Mazadiego ${ }^{4}$ \\ ${ }^{1}$ CNR-IGG - Istituto di Geoscienze e Georisorse, Pisa, Italy \\ ${ }^{2}$ Università di Firenze, Dipartimento di Scienze della Terra, Firenze, Italy \\ ${ }^{3}$ CNR-IGG - Istituto di Geoscienze e Georisorse, Firenze, Italy \\ ${ }^{4}$ Universidad Politécnica de Madrid, Escuela Técnica Superior de Ingenieros de Minas y Energía, Madrid, Spain \\ ${ }^{5}$ Unione dei Comuni Amiata-Val d'Orcia, Castiglion d'Orcia (Siena), Italy
}

\begin{abstract}
Article history
Received May 5, 2014; accepted August 15, 2014.

Subject classification:

Central Italy, Amiata volcano, Hydrothermal gases, $\mathrm{CO}_{2}$, Environmental impact.
\end{abstract}

\begin{abstract}
The Mt. Amiata volcano (Tuscany, central Italy) hosts the second largest geothermal field of Italy. Its $S W$ and NE sectors are characterized by the presence of several $\mathrm{CO}_{2}$-rich (>95\% by vol.) gas discharges. An intense $\mathrm{Hg}$ mining activity had taken place from the 19th century up to the end of the '70s, particularly close to Abbadia San Salvatore, during which two drillings (Acqua Passante and Ermeta) intercepted a $\mathrm{CO}_{2}$-rich gas fertile horizon. The related gases are emitted in the atmosphere since 1938 and 1959, respectively, causing severe concerns for the local air quality. In this work the results of a geochemical and isotopic survey carried out on these gas emissions from March 2009 to January 2014 are presented. $\mathrm{CO}_{2}$ fluxes from both the two wells and soil from an area of about 653,500 $\mathrm{m}^{2} \mathrm{lo}$ cated between them were measured. The two wells are emitting up to 15,000, 92 and 8 tons $y^{-1}$ of $\mathrm{CO}_{2}, \mathrm{CH}_{4}$ and $\mathrm{H}_{2} \mathrm{~S}$, respectively, while the computed soil $\mathrm{CO}_{2}$ output was estimated at 4,311 ton $y^{-1}$. The spatial distribution of the $\mathrm{CO}_{2}$ soil flux suggests the presence of preferential patterns, indicating sites of higher permeability. Since the local municipality is evaluating the possibility to plug the Ermeta vent, a temporarily closure should first be carried out to test the possible influence of this operation on the diffuse soil degassing of deep-originated $\mathrm{CO}_{2}$ in the surrounding area. This implies that diffuse soil gases should carefully be monitored before proceeding with its definitive closure.
\end{abstract}

\section{Introduction}

The occurrence of $\mathrm{CO}_{2}$-rich gas discharges is typical of active, quiescent and recent volcanic activity [e.g. Allard et al. 1991, Burton et al. 2013 and references therein]. Natural subsurface fluid reservoirs accumulating $\mathrm{CO}_{2}$ produced by magma degassing and/or thermo-metamorphic reactions of carbonates are indeed a relatively common geological feature, particularly in areas affected by subduction processes or lithospheric thinning where volcanic [e.g. Gerlach 1991] and/ or geothermal [e.g. Brombach et al. 2001, Chiodini et al. 2004a, 2005] systems occur. Carbon dioxide released from these reservoirs is discharged into the atmosphere through soil diffuse degassing and/or punctual gas manifestations [e.g. Chiodini et al. 1999, 2010, Rogie et al. 2001, Minissale 2004].

In the peri-Tyrrhenian Italy, a region characterized by geothermal systems (e.g. Larderello, Mt. Amiata) and Quaternary (Roman Co-magmatic Province) and present (e.g. Vesuvius, Phlegrean Fields, Aeolian Islands) volcanic activity related to crustal thinning generated by the opening of the Tyrrhenian Sea [e.g. Zitellini et al. 1986, Bartole 1995, Mauffret et al. 1999, Peccerillo 2003, 2005, Peccerillo and Lustrino 2005], a large amount of deep-originated $\mathrm{CO}_{2}$ is discharged from gas vents and diffuse soil emissions, and is present as dissolved inorganic carbon in ground waters [e.g. Minissale et al. 1997a, Chiodini et al. 1998, 2004b, Gambardella et al. 2004, Minissale 2004]. It has been estimated that $>25.000$ ton $\mathrm{CO}_{2}$ day $^{-1}$ are naturally discharged by the Tuscan-Roman and Campanian Degassing Systems (TRDS and CDS, respectively) [Chiodini et al. 2004a]. In these areas, $\mathrm{CO}_{2}$-rich (>90\% by volume) gases discharged from gas vents located in subcircular-shaped craters and associated to fissures, frac- 


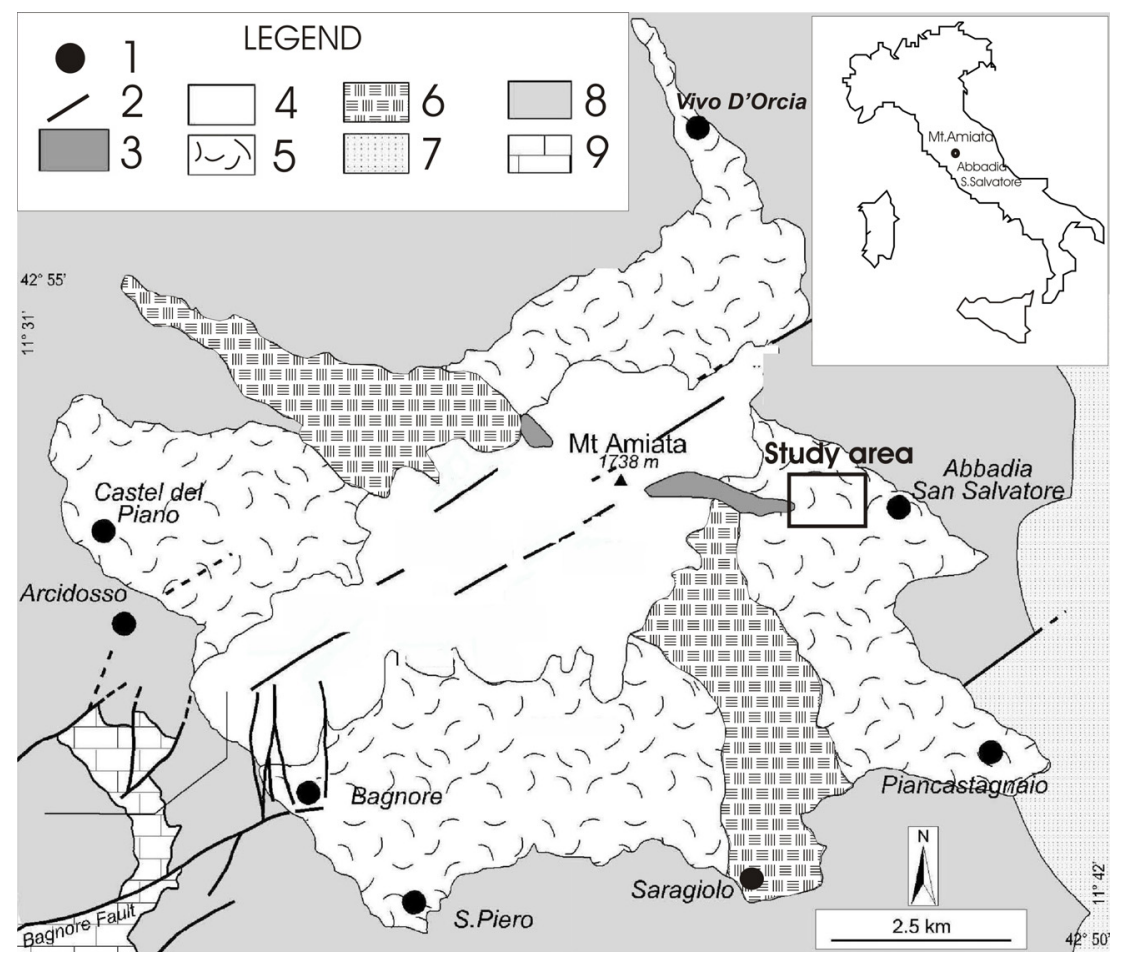

Figure 1. Geological sketch map of Mt. Amiata and location of the study area. Legend: (1) town; (2) fault; (3) third volcanic complex: the Ermeta olivine-latite lava flow: $209 \pm 0.29 \mathrm{ka}$; (4) second volcanic complex: porphyritic rhyodacite dome and lava flow, from $300 \pm 0.04 \mathrm{ka}$ to $190 \pm 0.23 \mathrm{ka}$; (5) first volcanic complex, upper unit: trachydacite: from $318 \pm 0.08 \mathrm{ka}$ to $217 \pm 0.01 \mathrm{ka}$; (6) first volcanic complex, lower unit: trachydacite: from $305 \pm 0.005$ ka to $241 \pm 0.07$ ka; (7) clay and sand: Early Pliocene; (8) Ligurian Units: Jurassic-Middle Eocene; (9) Tuscan Nappe: late Triassic-Oligocene (modified after Brogi et al. [2010]).

tures and structural lows (e.g. sinkholes), have caused fatal accidents, which have involved animals and human beings [e.g. Carapezza et al. 2003, Vaselli et al. 2006a, Tassi et al. 2009]. Locally, $\mathrm{CO}_{2}$ accumulation in air may be favored since this gas is relatively heavy (gas density of $\mathrm{CO}_{2}$ and air being of 0.001977 and $0.001280 \mathrm{~g} \mathrm{~mL}^{-1}$, respectively). As a consequence, when the climatic conditions are characterized by low wind, cloudy weather and cold air temperature, the morphologically depressed areas hosting these gas vents may become deadly traps. Such $\mathrm{CO}_{2}$-rich gas discharges are commonly accompanied by the presence of other toxic, dangerous and inflammable gases such as $\mathrm{H}_{2} \mathrm{~S}$ and/or $\mathrm{CH}_{4}$, whose contents can be up to a few percentages [e.g. Minissale et al. 1997a, Minissale 2004].

The Mt. Amiata volcanic edifice is located in southern Tuscany (Figure 1). It represents the biggest and youngest volcano $(\sim 200,000$ years old $)$ of this region and hosts the second largest geothermal field of Italy after Larderello. Its south-westernmost (e.g. Selvena, Banditella and Zancona; Minissale et al. [1997b], Frondini et al. [2009]) and east-northernmost (e.g. Bagni San Filippo and Pietrineri; Minissale et al. [1997b], Frondini et al. [2009], Tassi et al. [2009]) flanks are characterized by the presence of several $\mathrm{CO}_{2}$-rich ( $>95 \%$ by volume) gas discharges, mostly recognized during mercury (cinnabar) exploitation in the eastern part of this volcanic complex. In 1938 and 1959 two wells, named
Acqua Passante (1,048 a.s.1.) and Ermeta (1,079 m a.s.1.), were drilled close to the town of Abbadia San Salvatore (Figure 2) in the volcanic cover at the depth of 116 and $298 \mathrm{~m}$, respectively. It is worth to point out that, despite the large number of mining galleries, none of them was intercepted during the drilling activity. Currently, gases from these wells are discharged at the height of about $6 \mathrm{~m}$ above the ground, as two chimneys were built to minimize the disagreeable effects for the people approaching these areas due to the presence of $\mathrm{CO}_{2}$ and $\mathrm{H}_{2} \mathrm{~S}$, the latter having the typical smell of rotten eggs.

Once the $\mathrm{Hg}$ mining activity was terminated, the owner of the mining concession (E.N.I.: National Agency for Hydrocarbons, AGIP Division) produced a series of documentation for the reclamation of the $\mathrm{Hg}$ extraction and processing areas. Finally, in 2008, an agreement between E.N.I. and the municipality of Abbadia San Salvatore was signed and, consequently, the ownership of the reclamation passed to the public agency [e.g. Vaselli et al. 2013]. In the agreement the Ermeta and Acqua Passante wells were also mentioned, since the local municipality was in charge to: i) periodically monitor the discharged gases; ii) efficiently maintain the chimneys and iii) propose alternative strategies to reduce the impact of the discharged gases released to the atmosphere.

The Acqua Passante well is located close to a picnic area along the main road that leads to the top of $\mathrm{Mt}$. 


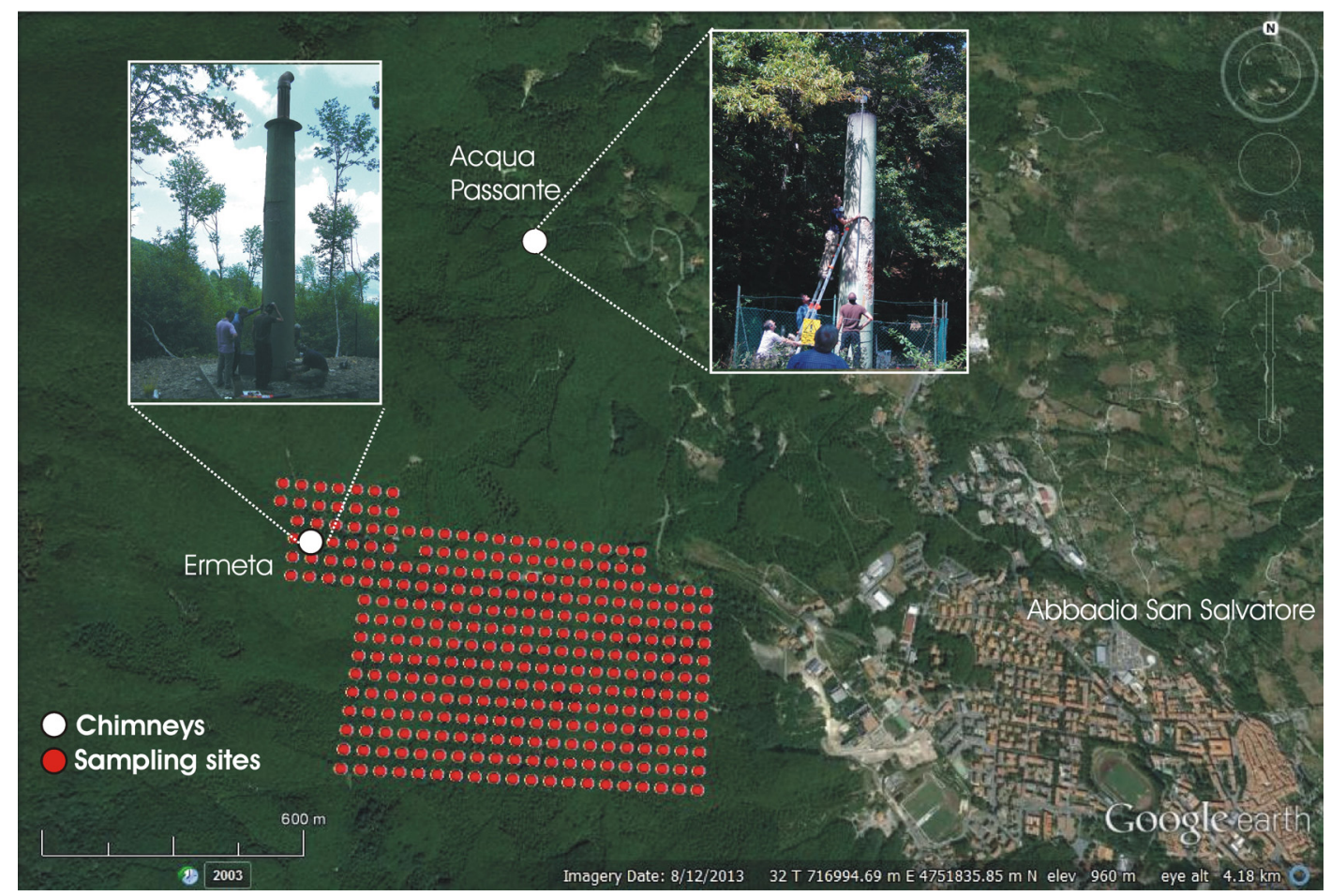

Figure 2. Location of the i) Ermeta and Acqua Passante wells and ii) soil diffuse $\varphi \mathrm{CO}_{2}$ measurement sites.

Amiata, where skiing and trekking facilities are present. That of Ermeta is close the homonymous church (built in 1296) that is an important tourist and religious site.

In this paper we present the results of a geochemical and isotopic survey (from March 2009 to January 2014) carried out on the gases discharged from these two chimneys. The $\mathrm{CO}_{2}$ flux from both the two vents and those from the soil carried out in July 2011 in an area of $653,550 \mathrm{~m}^{2}$ located SE of the Ermeta chimney (Figure 2), was measured by using the hot-wire anemometer and accumulation chamber method [Baubron et al. 1990, 1991], respectively. The chemical composition of the gas discharges located close to the study area [Tassi et al. 2009] was reported for comparison.

The main goals of this study were those to: i) investigate the genetic relationships between the well gases and those naturally discharging nearby and ii) verify the possible implications related to the closure of the Ermeta and Acqua Passante wells by the local municipality to reduce the impact of the discharged gases on the local population and tourists.

\section{Geological setting and mining activity}

The Mt. Amiata area (southern Tuscany, central Italy; Figure 1) is characterized by a volcanic activity that lasted ca. 100,000 years, the related deposits, dominated by a trachydacitic composition, being emplaced between 300 and $190 \mathrm{ka}$ [Ferrari et al. 1996, Cadoux and Pinti 2009]. The "residual" volcanic activity of this edifice related to the emplacement, at $6-7 \mathrm{~km}$ below the sea level, of a magmatic body in the Pliocene, produced two geothermal reservoirs, which are feeding a number of $\mathrm{CO}_{2}$-rich gas manifestations and $\mathrm{Ca}-\mathrm{SO}_{4}$-rich thermo-mineral waters, mainly located in NE and SW sector of the volcanic edifice [Minissale et al. 1997b, Frondini et al. 2009, Tassi et al. 2009]. Presently, two geothermal plants are operating at Bagnore and Piancastagnaio (Figure 1).

The geodynamic setting of the Mt. Amiata geothermal area is dominated by extensional structures developed from the Middle Miocene to the Quaternary, whilst collisional structures occurred from the Cretaceous to the Early Miocene [e.g. Brogi et al. 2010 and references therein]. The anomalous heat flux related to the Pliocene magmatic body, likely caused the emplacement of $\mathrm{Hg}$ ore deposits [Tanelli 1983, Klemm and Neumann 1984]. In the recent past, Mt. Amiata was one of the biggest $\mathrm{Hg}$ producers in the world [e.g. Rimondi et al. 2011, Vaselli et al. 2013 and references therein]. The Mt. Amiata Hg district is mainly distributed in the central-eastern and south-western sectors of this volcanic complex, although the principal mining activity was located in the municipality of Abbadia San Salvatore (Figure 1). The extraction and metallurgy of cinnabar (HgS) was aimed to produce metallic mercury from historic times until the middle seventies, when most of the $\mathrm{Hg}$ mines in the world were shut down due to environmental concerns. In the second half of the last century several wells, including those of Ermeta and Acqua Passante, were drilled for mining exploration close to Abbadia San Salvatore (Figure 2). It is worthwhile to mention that some of the mining gal- 
leries were affected by dangerous $\mathrm{CO}_{2}$ emissions, occasionally causing fatalities, and $\mathrm{CO}_{2}$ is still abundantly present in some of them [Vaselli et al. 2006a, 2011]. Both the Ermeta and Acqua Passante wells are located in the volcanic cover and that of Ermeta is situated few hundreds meters from the Ermeta olivine-latite lava flow that marked the latest event of the volcanic history of Mt. Amiata [e.g. Ferrari et al. 1996].

\section{Material and methods}

\subsection{Sampling and analysis of gases}

The Ermeta well is located to the east of Abbadia San Salvatore whilst that of Acqua Passante is about 1 $\mathrm{km} \mathrm{NE}$ on a fly of bird from Ermeta (Figure 2). From the two wells, 18 gas samples were collected from March 2009 to January 2014.

The well gases were collected using a $40 \mathrm{~cm}$ long Tygon ${ }^{\circledR}$ tube inserted into an artificial opening $(25 \mathrm{~mm}$ in diameter) located at the base of the two $\sim 6 \mathrm{~m}$ high chimneys (whose diameters are $300 \mathrm{~mm}$ ) and connected to a pre-evacuated $60 \mathrm{~mL}$ glass thorion-tapped flask filled with $20 \mathrm{~mL}$ of a $4 \mathrm{~N} \mathrm{NaOH}$ and $0.15 \mathrm{M}$ $\mathrm{Cd}(\mathrm{OH})_{2}$ suspension [Vaselli et al. 2006b and references therein]. The analysis of the inorganic gases stored in the headspace of the sampling flasks $\left(\mathrm{N}_{2}, \mathrm{O}_{2}, \mathrm{H}_{2}, \mathrm{He}\right.$, $\mathrm{CH}_{4}, \mathrm{Ar}$ and $\mathrm{Ne}$ ) was carried out with a Shimadzu 15A gas-chromatograph equipped with a $10 \mathrm{~m}$ long $5 \mathrm{~A}$ molecular sieve column and a Thermal Conductivity Detector (TCD). The alkaline solution, separated from the solid precipitate by centrifugation, was used for the determination of $\mathrm{CO}_{2}$, as $\mathrm{CO}_{3}^{2-}$, by titration (Metrohm Basic Titrino) with a $0.5 \mathrm{~N} \mathrm{HCl}$ solution. The solid CdS was oxidized to $\mathrm{SO}_{4}^{2-}$ with $\mathrm{H}_{2} \mathrm{O}_{2}$ and analyzed for the determination of $\mathrm{H}_{2} \mathrm{~S}$ by ion-chromatography [Montegrossi et al. 2001]. Analytical error was $<5 \%$. The ${ }^{13} \mathrm{C} /{ }^{12} \mathrm{C}$ isotopic ratio of $\mathrm{CO}_{2}$ (expressed as $\delta^{13} \mathrm{C}-\mathrm{CO}_{2} \%$ $\mathrm{V}-\mathrm{PDB}$ ) was measured after the purification and extraction of $\mathrm{CO}_{2}$ by using the two cryogenic traps method [Vaselli et al. 2006b]. The analytical error and the reproducibility were $\pm 0.05 \%$ ond $\pm 0.1 \%$ o, respectively. The determination of the ${ }^{3} \mathrm{He} /{ }^{4} \mathrm{He}$ ratios (expressed as $\mathrm{R} / \mathrm{R}_{\mathrm{a}}$, where $\mathrm{R}$ is the ${ }^{3} \mathrm{He} /{ }^{4} \mathrm{He}$ measured ratio and $\mathrm{R}_{\mathrm{a}}$ is the ${ }^{3} \mathrm{He} /{ }^{4} \mathrm{He}$ ratio in the air: $1.39^{\star} 10-6$; Mamyrin and Tolstikhin [1984]) was carried out at the University of Rochester Rare Gas Facility using a VG 5400 Rare Gas Mass Spectrometer by using the method described in Poreda and Farley [1992]. The measured ${ }^{3} \mathrm{He} /{ }^{4} \mathrm{He}$ ratios were corrected for the addition of air on the basis of the ${ }^{4} \mathrm{He} /{ }^{20} \mathrm{Ne}$ ratios measured by mass spectrometry, assuming that ${ }^{20} \mathrm{Ne}$ had an atmospheric origin [Craig and Lupton 1976, Sano and Wakita 1988]. Analytical error for $\mathrm{R} / \mathrm{R}_{\mathrm{a}}$ determination was $\leq 0.3 \%$.
3.2. Measurements of the $\mathrm{CO}_{2}$ flux from the chimneys and diffuse soil

The $\mathrm{CO}_{2}$ flux of the two chimneys was determined by inserting into the chimneys (whose section had an area of $0.07 \mathrm{~m}^{2}$ ) a Delta Ohm model HD2103.2 hot-wire anemometer probe. $\mathrm{CO}_{2}$ flux records were set to acquire the data from August 5 to September 5, 2011, during which the $\mathrm{CO}_{2}$ flux was measured every 5 minutes and was calculated on the basis of the discharged gas velocity and temperature inside the pipelines. The $\mathrm{CO}_{2}$ flux values were normalized at $20^{\circ} \mathrm{C}$. The temperature of the Ermeta chimney was ranging between 26.8 and $29^{\circ} \mathrm{C}$, while that of Acqua Passante was between 16.9 and $22.5^{\circ} \mathrm{C}$.

The survey of diffuse soil $\mathrm{CO}_{2}$ flux (July 2011) covered an area of $653,550 \mathrm{~m}^{2}$ with 301 evenly distributed measuring sites and was carried out in the SE sector of the Ermeta chimney (Figure 2). All the measurements were carried out where soils developed on the Mt. Amiata volcanic products, avoiding those areas where the volcanic rocks were cropping out.

To measure the $\mathrm{CO}_{2}$ flux from the soil, a direct and dynamic method that follows the principle of the socalled accumulation chamber [Baubron et al. 1990, 1991] was used. This method, initially used to determine soil respiration [i.e. Kanemasu et al. 1974, Parkinson 1981], has been widely applied for measuring diffuse soil $\mathrm{CO}_{2}$ flux in volcanic and geothermal [e.g. Tonani and Miele 1991, Chiodini et al. 1996, 1998, 2000, 2003, 2004a, Pancioli et al. 2009, Tassi et al. 2009] and solid waste disposal landfills [e.g. Raco et al. 2006, 2008, Moretti et al. 2007]. The instrument consisted of: i) an inverted chamber equipped with a system to mix the air in the chamber headspace (volume $=3.06 \times 10^{-3} \mathrm{~m}^{3}$ and area height $=$ $100 \mathrm{~mm}$ ), ii) an Infra-Red (IR) spectrophotometer with LICOR Li-820 sensor (measuring range of 0-20,000 ppm, accuracy of $4 \%$ of reading), iii) an analogue-digital (AD) converter and iv) a palmtop computer (PC). In order to prevent damages to both the pump and the $\mathrm{CO}_{2}$ detector, a magnesium perchlorate desiccant trap was placed between the outlet fitting of the accumulation chamber and the inlet of the PTFE Filter to adsorb soil humidity.

Once the accumulation chamber was positioned on the ground a low-flux pump $(20 \mathrm{~mL} \mathrm{~s}-1)$ continuously convoyed the soil gas from the chamber to the IR sensor. To minimize the disturbance effects due to changes of barometric conditions, the soil gas was reinjected into the chamber.

The $\mathrm{dC}_{\mathrm{CO}_{2}} / \mathrm{dt}$ values were computed in the field with the PC connected with the IR through the AD. The soil $\varphi \mathrm{CO}_{2}$ was calculated on the basis of the measured $\mathrm{CO}_{2}$ concentration increment inside the chamber over time ( $\mathrm{dC}_{\mathrm{CO}_{2}} / \mathrm{dt}$ ), according to the following equation: 


\begin{tabular}{|c|c|c|c|c|c|c|c|c|c|c|c|}
\hline Name & Date & $\mathrm{CO}_{2}$ & $\mathrm{H}_{2} \mathrm{~S}$ & $\mathrm{~N}_{2}$ & $\mathrm{CH}_{4}$ & $\mathrm{Ar}$ & $\mathrm{O}_{2}$ & $\mathrm{H}_{2}$ & $\mathrm{He}$ & $\delta^{13} \mathrm{C}-\mathrm{CO}_{2}$ & $\mathrm{R} / \mathrm{Ra}$ \\
\hline Ermeta & Mar-09 & 986 & 0.30 & 2.8 & 10 & 0.014 & 0.16 & 0.074 & 0.00064 & n.d. & 0.33 \\
\hline Ermeta & Jul-09 & 982 & 0.77 & 14 & 2.8 & 0.048 & 0.057 & 0.078 & 0.00097 & -3.39 & \\
\hline Ermeta & Oct-09 & 978 & 0.62 & 15 & 5.7 & 0.025 & 0.004 & 0.68 & 0.0013 & -3.39 & \\
\hline Ermeta & Dec-09 & 986 & 0.56 & 4.2 & 8.9 & 0.038 & 0.23 & 0.12 & 0.0012 & -3.33 & \\
\hline Ermeta & Apr-09 & 986 & 0.36 & 5.1 & 7.7 & 0.056 & 0.16 & 0.21 & 0.0014 & -3.35 & \\
\hline Ermeta & Jul-10 & 987 & 0.33 & 5.5 & 6.9 & 0.053 & 0.19 & 0.12 & 0.00089 & -3.48 & 0.32 \\
\hline Ermeta & Oct-10 & 987 & 0.40 & 6.2 & 5.8 & 0.045 & 0.16 & 0.24 & 0.00055 & -3.13 & \\
\hline Ermeta & Nov-10 & 987 & 0.33 & 6.3 & 5.6 & 0.049 & 0.16 & 0.21 & 0.00069 & -3.27 & \\
\hline Ermeta & Apr-11 & 989 & 0.58 & 4.6 & 5.5 & 0.032 & 0.14 & 0.54 & 0.00091 & -3.27 & \\
\hline Ermeta & Jun-11 & 987 & 0.42 & 5.6 & 6.7 & 0.037 & 0.15 & 0.55 & 0.00075 & -3.41 & \\
\hline Ermeta & Oct-11 & 983 & 0.77 & 6.1 & 9.5 & 0.045 & 0.14 & 0.44 & 0.00074 & -3.45 & \\
\hline Ermeta & Dec-11 & 987 & 0.34 & 5.6 & 6.6 & 0.056 & 0.19 & 0.25 & 0.00066 & -3.09 & \\
\hline Ermeta & Mar-12 & 988 & 0.55 & 5.3 & 5.1 & 0.052 & 0.21 & 0.34 & 0.00039 & -3.33 & \\
\hline Ermeta & Apr-12 & 989 & 0.45 & 5.2 & 5.0 & 0.046 & 0.21 & 0.28 & 0.00031 & n.d. & \\
\hline Ermeta & Jul-12 & 988 & 0.52 & 5.6 & 5.0 & 0.056 & 0.15 & 0.30 & 0.00028 & -3.36 & \\
\hline Ermeta & Jan-13 & 989 & 0.72 & 5.1 & 4.9 & 0.052 & 0.19 & 0.26 & 0.00018 & -3.27 & \\
\hline Ermeta & Jul-13 & 989 & 0.81 & 5.0 & 4.6 & 0.061 & 0.22 & 0.27 & 0.00011 & -3.32 & \\
\hline Ermeta & Jan-14 & 989 & 0.66 & 5.5 & 4.8 & 0.036 & 0.16 & 0.22 & 0.00016 & & \\
\hline Acqua Passante & Mar-09 & 987 & 0.34 & 6.4 & 5.9 & 0.029 & 0.11 & 0.080 & 0.00069 & -3.31 & 0.29 \\
\hline Acqua Passante & Jul-09 & 977 & 0.33 & 12 & 11 & 0.043 & 0.73 & 0.069 & 0.0022 & -2.92 & \\
\hline Acqua Passante & Oct-09 & 988 & 0.30 & 7.5 & 4.0 & 0.020 & 0.01 & 0.12 & 0.00063 & -3.38 & \\
\hline Acqua Passante & Dec-09 & 988 & 0.39 & 6.1 & 5.0 & 0.032 & 0.16 & 0.085 & 0.00087 & -3.33 & \\
\hline Acqua Passante & Apr-09 & 988 & 0.22 & 6.7 & 4.5 & 0.063 & 0.22 & 0.076 & 0.00061 & -3.31 & \\
\hline Acqua Passante & Jul-10 & 988 & 0.22 & 6.4 & 5.2 & 0.060 & 0.20 & 0.087 & 0.00055 & -3.47 & 0.40 \\
\hline Acqua Passante & Oct-10 & 989 & 0.32 & 5.9 & 4.4 & 0.053 & 0.13 & 0.18 & 0.00087 & -3.26 & \\
\hline Acqua Passante & Nov-10 & 988 & 0.36 & 6.1 & 4.9 & 0.056 & 0.11 & 0.22 & 0.00065 & -3.13 & \\
\hline Acqua Passante & Apr-11 & 989 & 0.59 & 5.2 & 5.0 & 0.036 & 0.14 & 0.39 & 0.00086 & -3.13 & \\
\hline Acqua Passante & Jun-11 & 987 & 0.37 & 5.7 & 6.0 & 0.041 & 0.16 & 0.34 & 0.00061 & -3.11 & \\
\hline Acqua Passante & Oct-11 & 985 & 0.66 & 6.4 & 7.8 & 0.030 & 0.12 & 0.33 & 0.00066 & -3.39 & \\
\hline Acqua Passante & Dec-11 & 986 & 0.30 & 6.3 & 6.6 & 0.061 & 0.27 & 0.14 & 0.00044 & -3.25 & \\
\hline Acqua Passante & Mar-12 & 989 & 0.40 & 5.5 & 5.0 & 0.061 & 0.24 & 0.26 & 0.00035 & -2.84 & \\
\hline Acqua Passante & Apr-12 & 989 & 0.41 & 5.4 & 5.1 & 0.053 & 0.19 & 0.23 & 0.00025 & n.d. & \\
\hline Acqua Passante & Jul-12 & 989 & 0.45 & 5.3 & 4.9 & 0.055 & 0.18 & 0.25 & 0.00024 & -3.33 & \\
\hline Acqua Passante & Jan-13 & 989 & 0.56 & 5.1 & 4.6 & 0.056 & 0.22 & 0.25 & 0.00015 & -3.21 & \\
\hline Acqua Passante & Jul-13 & 989 & 0.63 & 5.1 & 4.8 & 0.050 & 0.19 & 0.31 & 0.00013 & -3.35 & \\
\hline Acqua Passante & Jan-14 & 989 & 0.58 & 5.3 & 4.9 & 0.054 & 0.18 & 0.26 & 0.00019 & & \\
\hline Name & ID & $\mathrm{CO}_{2}$ & $\mathrm{H}_{2} \mathrm{~S}$ & $\mathrm{~N}_{2}$ & $\mathrm{CH}_{4}$ & $\mathrm{Ar}$ & $\mathrm{O}_{2}$ & $\mathrm{H}_{2}$ & $\mathrm{He}$ & $\delta^{13} \mathrm{C}-\mathrm{CO}_{2}$ & $\mathrm{R} / \mathrm{Ra}$ \\
\hline Polla nera ${ }^{\star}$ & $\mathrm{PN}$ & 980 & 0.54 & 11 & 8.7 & 0.025 & 0.0047 & 0.0020 & 0.0031 & & \\
\hline Spuntone ${ }^{\star}$ & SP & 972 & 0.55 & 16 & 11 & 0.032 & 0.0027 & 0.00003 & 0.0045 & -2.87 & \\
\hline Hole $^{\star}$ & $\mathrm{HO}$ & 982 & 0.61 & 11 & 6.0 & 0.021 & 0.0020 & 0.0014 & 0.0033 & -2.61 & \\
\hline Poggio all'Olivo* & $\mathrm{PO}$ & 983 & 0.95 & 8.6 & 7.2 & 0.018 & 0.0038 & 0.00043 & 0.0026 & -3.24 & \\
\hline Polla Grigia & PG & 976 & 0.79 & 13 & 11 & 0.029 & 0.0028 & 0.0034 & 0.0039 & & \\
\hline Mammellone ${ }^{\star}$ & MA & 978 & 0.81 & 14 & 7.5 & 0.022 & 0.0021 & 0.00057 & 0.00086 & -2.63 & 0.13 \\
\hline
\end{tabular}

Table 1. Chemical (in $\mathrm{mmol} / \mathrm{mol}$ ) and isotopic (carbon: as $\delta^{13} \mathrm{C}-\mathrm{CO}_{2}$ vs. V-PDB \%; and helium as R/Ra) composition of the Ermeta and Acqua Passante gases and of the bubbling pools and dry vents (PN, SP HO, PO, PG and MA). ( ${ }^{\star}$ ): data from Tassi et al. [2009]. 


$$
\varphi \mathrm{CO}_{2}=c f \times\left(d C_{\mathrm{CO}_{2}} / d t\right)
$$

where the proportionality factor ( $c f$ ) between $d_{C_{2}} / d t$ and $\varphi \mathrm{CO}_{2}$ was determined by laboratory tests through an artificial soil, during which an interval of controlled $\varphi \mathrm{CO}_{2}$ values (from 10 to $10,000 \mathrm{~g} \mathrm{~m}^{-2}$ day $^{-1}$ ) was measured (at least 6 measurements were repeated for each $\varphi \mathrm{CO}_{2}$ value). The $c f$ factor was then computed as the slope of the linear best-fit line of $\varphi \mathrm{CO}_{2}$ vs. $d C_{\mathrm{CO}_{2}} / d t$.

\section{Results}

4.1. Chemical and isotopic $\left(\delta^{13} \mathrm{C}-\mathrm{CO}_{2}\right.$ and $\left.\mathrm{R} / \mathrm{R}_{a}\right)$ compositions of gases from wells, bubbling pools and dry vents

The chemical and isotopic $\left(\delta^{13} \mathrm{C}-\mathrm{CO}_{2}\right.$ and $\mathrm{R} / \mathrm{R}_{\mathrm{a}}$ values) compositions of the Ermeta and Acqua Passante gases and, for comparison, those of six bubbling pools and dry gas vents (namely PN, SP HO, PO, PG and MA; see Tassi et al. [2009] for their location), located at about $5 \mathrm{~km}$ NE of Abbadia San Salvatore, are reported in Table 1.

The Ermeta and Acqua Passante wells discharged gases having a similar composition, being both dominated by $\mathrm{CO}_{2}$ (up to $989 \mathrm{mmol} \mathrm{mol}^{-1}$ ), with significant concentrations of $\mathrm{N}_{2}$ (up to 14 and $12 \mathrm{mmol} \mathrm{mol}^{-1}$, respectively), $\mathrm{CH}_{4}$ (up to 10 and $11 \mathrm{mmol} \mathrm{mol}^{-1}$, respectively) and $\mathrm{H}_{2} \mathrm{~S}$ (up to 0.81 and $0.66 \mathrm{mmol} \mathrm{mol}^{-1}$, respectively). Hydrogen concentrations ranged from 0.069 (Acqua Passante, July 2009) to $0.55 \mathrm{mmol} \mathrm{mol}^{-1}$ (Ermeta, June 2011), while those of noble gases, i.e. Ar, He and $\mathrm{Ne}$, were up to $0.061,0.00022$, and $0.00003, \mathrm{mmol}$ $\mathrm{mol}^{-1}$, respectively. The chemical composition of the bubbling pools was basically identical to that of the two wells, with the only exception of $\mathrm{H}_{2}$, whose concentration in the well gases was up to 3 orders of magnitude lower than in the wells. Helium $(\mathrm{R} / \mathrm{Ra})$ and carbon $\left(\delta^{13} \mathrm{C}^{-} \mathrm{CO}_{2}\right)$ isotopic compositions in the wells were in a narrow range, from 0.32 to 0.40 and from -3.48 to $-2.84 \%$ vs. V-PDB, respectively. The helium isotopic ratio for the natural gas discharges was only available for MA, whose value was moderately lower $(\mathrm{R} / \mathrm{Ra}=0.13)$ that those of the Ermeta and Acqua Passante gases, while those of carbon in $\mathrm{CO}_{2}$ were, on average, slightly more positive (between -3.24 and $-2.61 \%$ vs. V-PDB).

\section{2. $\mathrm{CO}_{2}$ flux from the chimneys and diffuse soil}

The database of the $\mathrm{CO}_{2}$ flux measurements from the Ermeta and Acqua Passante chimneys consisted of more than 9,000 measurements (not reported) and ranged from 8.86 to 11.06 (mean value: $10.05 \pm 0.45$ ) $\mathrm{mol} \mathrm{sec}^{-1}$ and from 0.89 to 2.51 (mean value: $1.32 \pm 0.17$ ) mol sec ${ }^{-1}$, respectively. The calculated $\mathrm{CO}_{2}$ output from Ermeta was $\approx 13,000$ ton $\mathrm{y}^{-1}$, whereas that of Acqua Passante clustered around 1,800 ton $\mathrm{y}^{-1}$.
The spatial distribution of the soil diffuse $\mathrm{CO}_{2}$ flux measurements was partly dictated by the rough and intensely vegetated territory. The measured $\varphi \mathrm{CO}_{2}$ values (301 data), ranging from 0.07 to $3.42 \mathrm{~mol} \mathrm{~m}^{-2} \mathrm{day}^{-1}$ with an average value of $0.38 \mathrm{~mol} \mathrm{~m}^{-2}$ day $^{-1}$ (Table 2), showed a normal logarithmic behavior with a 0.05 significance level (Figure 3), indicating that the $\ln \left(\varphi \mathrm{CO}_{2}\right)$ values had a modal density distribution. Mean, median, minimum, maximum $\varphi \mathrm{CO}_{2}$ values and standard deviation are reported as ln-values in Table 3.

\section{Discussion}

\subsection{Origin of gases}

The chemical composition of the Ermeta and Acqua Passante gases is basically consistent with that shown by the gas discharges located NE of Mt. Amiata, which are likely fed by a hydrothermal system consisting of two aquifers at distinct depth (500-1,000 $\mathrm{m}$ and $>3,000 \mathrm{~m}$, respectively) and temperature (up to 230 and $350^{\circ} \mathrm{C}$, respectively) [Calamai et al. 1970, Gianelli et al. 1988]. The $\delta^{13} \mathrm{C}^{-} \mathrm{CO}_{2}$ values (Table 1 ) are slightly heavier than the typical range of mantle $\mathrm{CO}_{2}$ [Rollinson 1993, Hoefs 1997, Ohmoto and Goldhaber 1997], suggesting that $\mathrm{CO}_{2}$ has a twofold origin, i.e. mantle degassing and thermometamorphic reactions acting on limestone [Minissale et al. 1997a, Frondini et al. 2009]. This feature is common to the great majority of the gas emissions from the peri-Tyrrhenian central Italy (Tuscan-Roman Degassing System, TRDS; Chiodini et al. [2004a]). In this region, a significant mantle contamination by crustal fluids released from the subducted Adriatic plate occurs [Martelli et al. 2004, Frondini et al. 2009], as testified by the relatively low $\mathrm{R} / \mathrm{Ra}$ values $(<0.4$; Table 1$)$. Thermogenic processes involving organic material buried in sedimentary formation are the most likely source for the high $\mathrm{N}_{2} /$ Ar ratios and $\mathrm{CH}_{4}$ and $\mathrm{H}_{2} \mathrm{~S}$ concentrations measured in gases from both the two wells and the nearby gas discharges. The relatively high $\mathrm{H}_{2}$ concentrations characterizing the Ermeta and Acqua Passante gases, significantly higher than those of the Mt. Amiata gas discharges (Figure 4), are likely resulting by the corrosion of the internal iron tubing of the wells, which is favored by the presence of $\mathrm{CO}_{2}$ - and $\mathrm{H}_{2} \mathrm{~S}$-rich gases. It is likely that this alteration process allows air to enter the chimneys. This would also explain the Ar enrichment shown by the wells gases with respect to the naturally discharges ones (Figure 5).

\subsection{Spatial variation and output of soil diffuse $\varphi \mathrm{CO}_{2}$}

In order to better constrain the total $\varphi \mathrm{CO}_{2}$ and visualize its spatial distribution, the $\mathrm{CO}_{2}$ iso-flux map is reported in Figure 6, which was drawn by means of the 


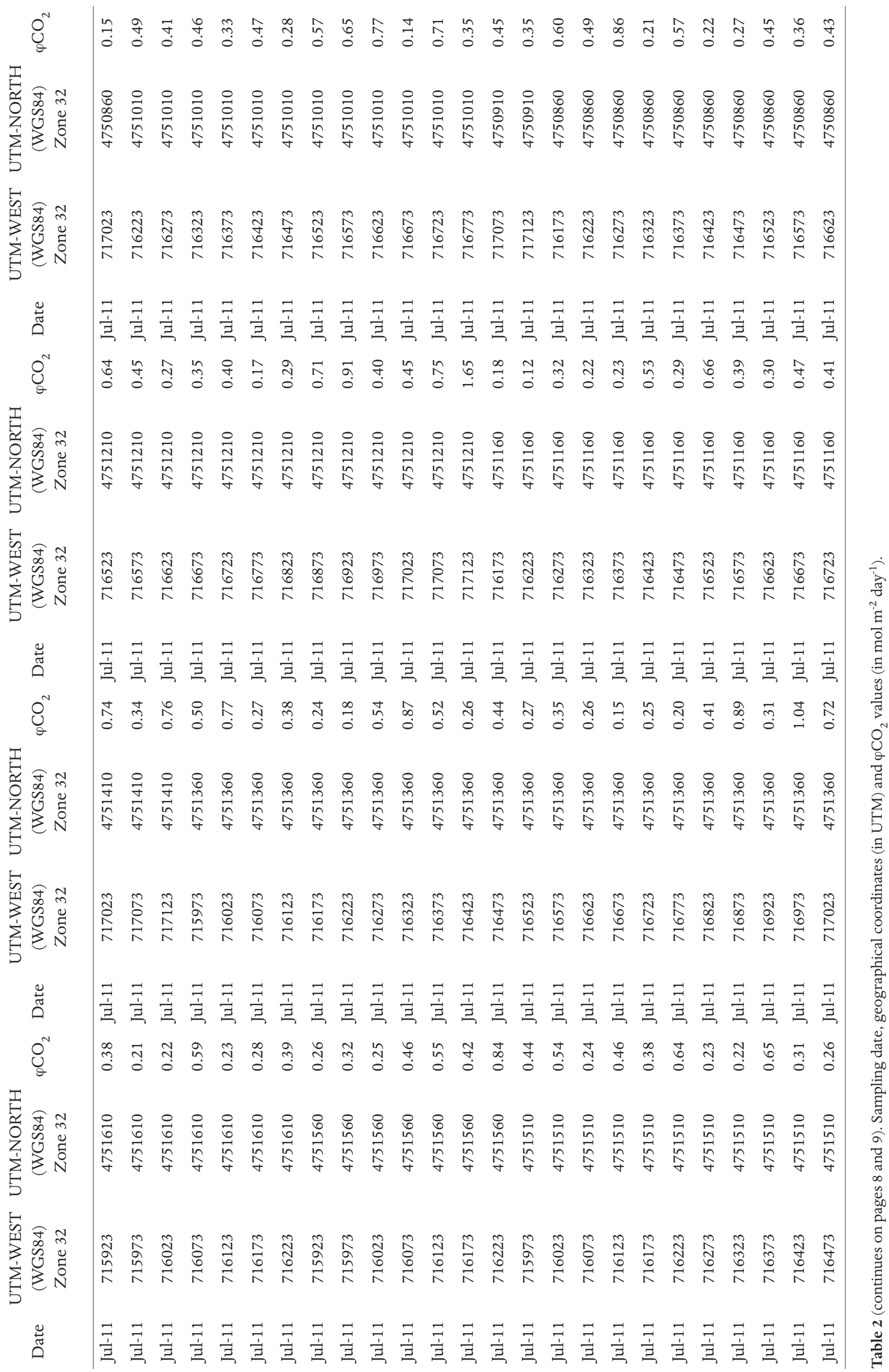




\begin{tabular}{|c|c|c|c|c|c|c|c|c|c|c|c|c|c|c|c|c|c|c|c|c|c|c|c|c|c|}
\hline 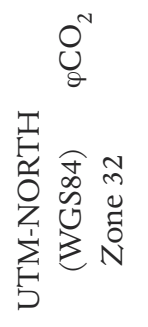 & 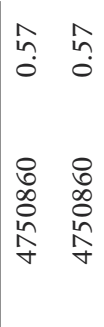 & 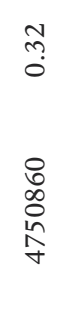 & 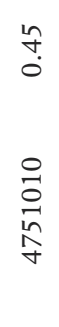 & 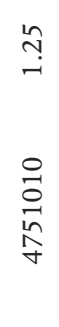 & $\begin{array}{l}\stackrel{0}{0} \\
\stackrel{0}{n} \\
\stackrel{n}{\sigma}\end{array}$ & $\begin{array}{l}\stackrel{0}{0} \\
\stackrel{0}{\vec{n}} \\
\stackrel{n}{f} \\
\text { fon }\end{array}$ & $\begin{array}{l}\stackrel{0}{0} \\
\stackrel{0}{n} \\
\stackrel{f}{\sigma}\end{array}$ & 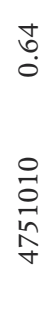 & $\begin{array}{l}\stackrel{0}{0} \\
\stackrel{0}{n} \\
\stackrel{n}{f}\end{array}$ & 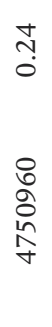 & $\stackrel{\swarrow}{\stackrel{n}{f}}$ & $\stackrel{\text { Ln }}{\stackrel{5}{f}}$ & h & 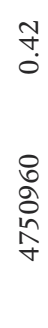 & $\stackrel{\gamma}{\digamma}$ & 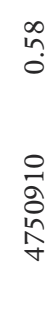 & & & & & & 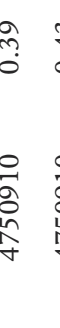 & $\dot{0}$ & & 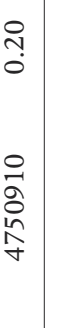 \\
\hline 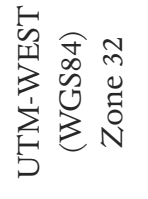 & 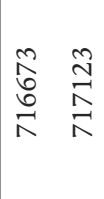 & 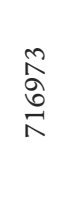 & 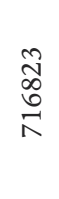 & $\begin{array}{l}\stackrel{n}{\Lambda} \\
\infty \\
\stackrel{\Lambda}{\Lambda}\end{array}$ & 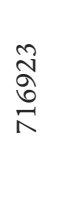 & $\begin{array}{l}\text { ñ } \\
\hat{\hat{o}} \\
\stackrel{1}{\Lambda}\end{array}$ & $\begin{array}{l}\text { ָे } \\
\stackrel{1}{\kappa}\end{array}$ & 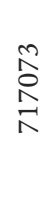 & 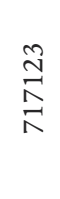 & $\begin{array}{l}\stackrel{m}{\hat{0}} \\
\stackrel{1}{\Lambda}\end{array}$ & 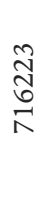 & $\begin{array}{l}\text { ते } \\
\stackrel{R}{\wedge}\end{array}$ & 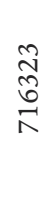 & 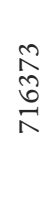 & 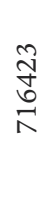 & f & & & $\approx$ & to & & 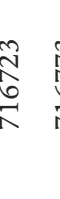 & 6 & 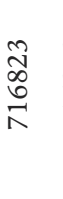 & 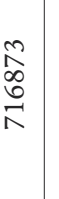 \\
\hline ڤँ & 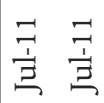 & $\frac{\exists}{3}$ & $\frac{\Xi}{\Xi}$ & $\frac{\Xi}{\Xi}$ & $\vec{\Xi}$ & $\frac{\Xi}{\Xi}$ & $\frac{\Xi}{\Xi}$ & $\frac{\exists}{\Xi}$ & $\frac{\Xi}{\Xi}$ & $\underset{\Xi}{\Xi}$ & $\frac{\Xi}{\Xi}$ & $\frac{\bar{I}}{\Xi}$ & $\exists$ & $I$ & & & & & & & & & $I$ & & $\frac{\Xi}{\Xi}$ \\
\hline$\bigcup_{\circlearrowleft}^{N}$ & $\begin{array}{cc}\infty & n \\
0 & n \\
0 & 0\end{array}$ & $\stackrel{\leftrightarrow}{\rightarrow}$ & $\stackrel{\overrightarrow{0}}{-}$ & $\stackrel{\text { In }}{\stackrel{0}{0}}$ & $\stackrel{0}{0}$ & $\begin{array}{l}\infty \\
\infty \\
0 \\
0\end{array}$ & $\stackrel{゚}{\stackrel{2}{-}}$ & तે & $\begin{array}{l}\stackrel{0}{0} \\
0\end{array}$ & กิ & $\stackrel{\infty}{0}$ & $\vec{b}$ & $\stackrel{-}{0}$ & $\stackrel{\infty}{\overbrace{0}^{0}}$ & $\stackrel{\circ}{0}$ & $\stackrel{a}{0}$ & & 1 & $\tilde{\alpha}$ & ? & h & $\begin{array}{l}n \\
\tilde{n} \\
0\end{array}$ & है & & $\begin{array}{c}\hat{y} \\
\text { ọ }\end{array}$ \\
\hline 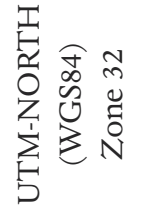 & 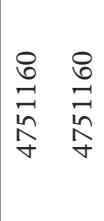 & $\begin{array}{l}\stackrel{8}{0} \\
\stackrel{7}{1} \\
\hat{f}\end{array}$ & 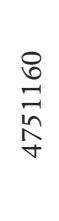 & 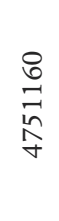 & $\begin{array}{l}\stackrel{8}{0} \\
\vec{n} \\
\hat{n} \\
\forall\end{array}$ & $\begin{array}{l}\stackrel{8}{7} \\
\overrightarrow{\mid} \\
\stackrel{n}{\sigma}\end{array}$ & 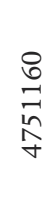 & 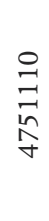 & 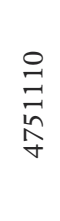 & 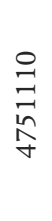 & 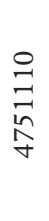 & 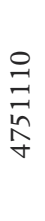 & 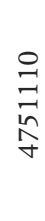 & \begin{tabular}{l}
$\stackrel{\vartheta}{\exists}$ \\
\multirow{2}{\sigma}{} \\
$f$
\end{tabular} & $\begin{array}{l}\vec{n} \\
\stackrel{n}{f}\end{array}$ & $\stackrel{ }{7}$ & & $\frac{5}{n}$ & 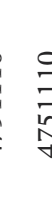 & 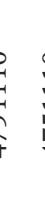 & & 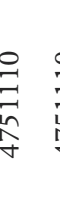 & $\begin{array}{l}\text { b } \\
\frac{\hbar}{f}\end{array}$ & 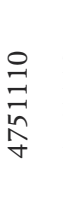 & 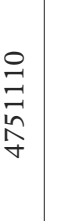 \\
\hline 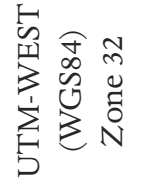 & 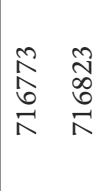 & $\begin{array}{l}\stackrel{n}{0} \\
\infty \\
\stackrel{\Lambda}{\Lambda}\end{array}$ & 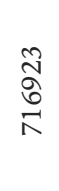 & $\begin{array}{l}\stackrel{n}{\hat{a}} \\
\stackrel{ }{\wedge}\end{array}$ & 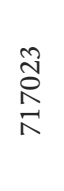 & 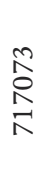 & $\underset{\stackrel{\widetilde{I}}{\mathbb{N}}}{\stackrel{R}{R}}$ & $\begin{array}{l}\stackrel{M}{\vec{b}} \\
\stackrel{ }{\Lambda}\end{array}$ & $\begin{array}{l}\text { స్ } \\
\stackrel{్}{K}\end{array}$ & $\begin{array}{l}\stackrel{\Re}{\hat{N}} \\
\stackrel{\Lambda}{\Lambda}\end{array}$ & 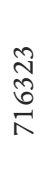 & 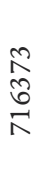 & 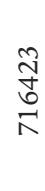 & 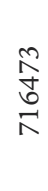 & 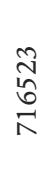 & 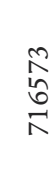 & & $\underline{v}$ & $\begin{array}{l}\hat{\text { }} \\
\text { î }\end{array}$ & th & & $\begin{array}{l}\text { त्र } \\
\text { o } \\
0 \\
\wedge \\
\end{array}$ & 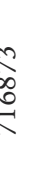 & 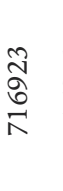 & 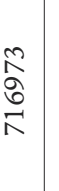 \\
\hline هٓ & $\begin{array}{ll}\bar{\Xi} & \bar{\Xi} \\
\Xi & \end{array}$ & $\exists$ & $\vec{\Xi}$ & $\frac{\Xi}{\Xi}$ & $\vec{\Xi}$ & $\exists$ & $\bar{\Xi}$ & $\exists$ & $\frac{\vec{\Xi}}{\Xi}$ & $\exists$ & $\exists$ & $\exists$ & $\exists$ & $\exists$ & & & & & & & & & & & $\exists$ \\
\hline$\stackrel{\circlearrowright}{\sigma}_{\sigma}^{N}$ & 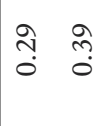 & $\overrightarrow{0}$ & f & $\stackrel{0}{\stackrel{0}{0}}$ & $\stackrel{\overbrace{}}{\stackrel{n}{\rightarrow}}$ & $\tilde{o}$ & $\stackrel{m}{0}$ & $\stackrel{ה}{\hat{0}}$ & $\hat{n}$ & $\overrightarrow{0}$ & ڤ̊ & חֶ & $\stackrel{?}{\circ}$ & $\stackrel{\overbrace{}}{ت}$ & in & 7 & & $\approx$ & s & q & ?े & $\frac{m}{0}$ & ? & $\begin{array}{l}\Omega \\
0 \\
0\end{array}$ & ঙ̣ \\
\hline 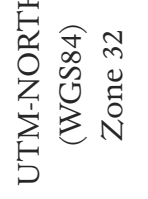 & 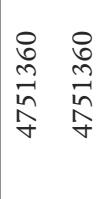 & 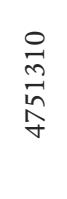 & $\begin{array}{l}\stackrel{0}{2} \\
\stackrel{n}{n} \\
\stackrel{n}{\hat{\sigma}}\end{array}$ & $\begin{array}{l}\stackrel{0}{\vec{m}} \\
\stackrel{n}{n} \\
\stackrel{n}{f}\end{array}$ & $\begin{array}{l}\stackrel{ }{\vec{n}} \\
\hat{n} \\
\hat{\sigma}\end{array}$ & \begin{tabular}{l}
$\stackrel{\circ}{\vec{n}}$ \\
$\vec{n}$ \\
\multirow{\sigma}{\sigma}{}
\end{tabular} & $\begin{array}{l}\stackrel{ }{\vec{m}} \\
\vec{n} \\
\stackrel{n}{f}\end{array}$ & $\begin{array}{l}\stackrel{ }{\vec{m}} \\
\stackrel{n}{\hat{n}} \\
\text { f }\end{array}$ & $\begin{array}{l}\stackrel{0}{\vec{m}} \\
\stackrel{n}{n} \\
\stackrel{f}{f}\end{array}$ & 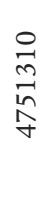 & $\begin{array}{l}\stackrel{0}{\vec{n}} \\
\stackrel{n}{\hat{\sigma}} \\
\stackrel{f}{r}\end{array}$ & 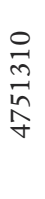 & 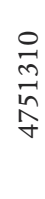 & 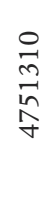 & $\begin{array}{l}\stackrel{0}{m} \\
\stackrel{n}{n} \\
\stackrel{n}{f}\end{array}$ & $\begin{array}{l}\stackrel{\circ}{a} \\
\stackrel{n}{n} \\
\stackrel{n}{f}\end{array}$ & & 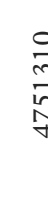 & $\frac{c}{3}$ & f & & 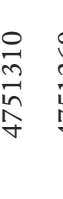 & $\begin{array}{l}\frac{b}{6} \\
\frac{1}{f} \\
\text { f }\end{array}$ & 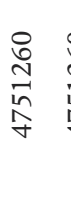 & 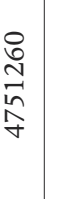 \\
\hline 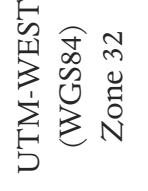 & 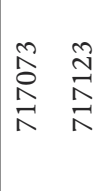 & $\begin{array}{l}\stackrel{N}{\overrightarrow{0}} \\
\vec{\Lambda}\end{array}$ & $\begin{array}{l}\text { त̦ } \\
\underset{\Lambda}{\mathbb{N}}\end{array}$ & 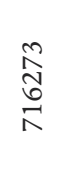 & 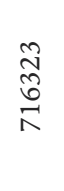 & 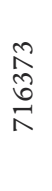 & $\begin{array}{l}\tilde{I} \\
\underset{\mathbb{Z}}{\mathbb{N}}\end{array}$ & 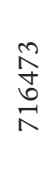 & 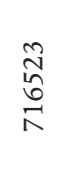 & $\begin{array}{l}n \\
\hat{2} \\
\stackrel{1}{1} \\
\end{array}$ & 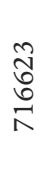 & 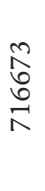 & 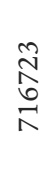 & $\begin{array}{l}\stackrel{N}{\hat{D}} \\
\stackrel{R}{R}\end{array}$ & శ్ & 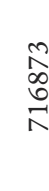 & $N$ & . & 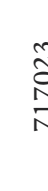 & S & 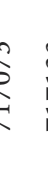 & $\begin{array}{l}\stackrel{7}{\pi} \\
\stackrel{\Lambda}{\Lambda}\end{array}$ & $\frac{n}{6}$ & 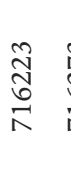 & 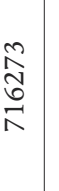 \\
\hline ص̆ & 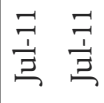 & $\frac{\bar{I}}{\Xi}$ & $\frac{\bar{I}}{\Xi}$ & $\frac{\exists}{\Xi}$ & $\frac{\vec{I}}{\Xi}$ & $\frac{\vec{I}}{\Xi}$ & $\vec{\Xi}$ & $\frac{\vec{E}}{\Xi}$ & $\frac{\Xi}{\Xi}$ & $\underset{\Xi}{\Xi}$ & $\frac{\vec{I}}{\Xi}$ & $\frac{\overline{1}}{\Xi}$ & $\exists$ & $\exists$ & & 7 & & & & & & $\frac{7}{\Xi}$ & $\frac{1}{3}$ & & $\frac{\bar{I}}{\Xi}$ \\
\hline$\stackrel{O}{O}_{\mathscr{\sigma}}^{N}$ & $\begin{array}{cc}\text { fr } & \text { n. } \\
0 & 0\end{array}$ & $\stackrel{\infty}{\infty}$ & $\stackrel{\text { \ุ }}{0}$ & $\stackrel{\text { J }}{0}$ & $\stackrel{I}{\exists}$ & 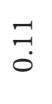 & $\begin{array}{l}\text { + } \\
0\end{array}$ & ?ֶ. & 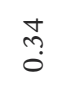 & 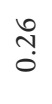 & $\approx$ & 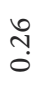 & $\underset{f}{+}$ & $\begin{array}{l}\infty \\
\text { in } \\
0\end{array}$ & & $\stackrel{+}{\sim}$ & & & is & t. & t & 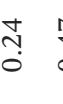 & y & & n? \\
\hline 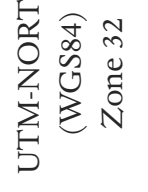 & 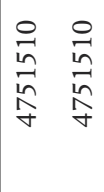 & $\begin{array}{l}\stackrel{ }{2} \\
i n \\
\hat{n} \\
\hat{f}\end{array}$ & 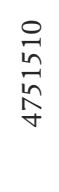 & 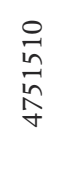 & 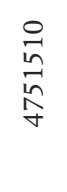 & 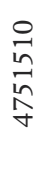 & 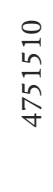 & 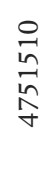 & 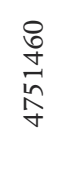 & 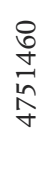 & 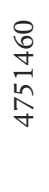 & 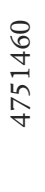 & \begin{tabular}{l}
$\stackrel{8}{0}$ \\
$\stackrel{+}{n}$ \\
\multirow{r}{f}{}
\end{tabular} & \begin{tabular}{l}
$\stackrel{8}{o}$ \\
$\stackrel{+}{n}$ \\
\multirow{\sigma}{f}{}
\end{tabular} & $\stackrel{8}{\square}$ & \begin{tabular}{l}
8 \\
$\stackrel{0}{0}$ \\
\multirow{2}{*}{} \\
\multirow{f}{*}{}
\end{tabular} & & 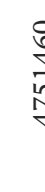 & 列 & ? & $\begin{array}{l}0 \\
\vdots \\
\vdots \\
f \\
f\end{array}$ & 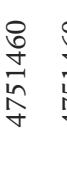 & $\frac{8}{d}$ & & 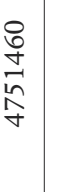 \\
\hline 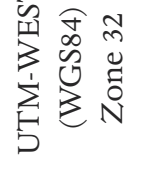 & 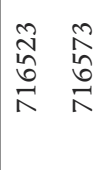 & $\begin{array}{l}\text { त్ర } \\
\stackrel{0}{\circ} \\
\end{array}$ & 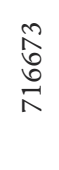 & 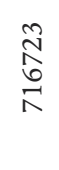 & 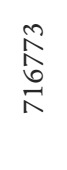 & $\begin{array}{l}\widetilde{N} \\
\infty \\
\stackrel{\Lambda}{\Lambda}\end{array}$ & $\begin{array}{l}\stackrel{n}{\infty} \\
\infty \\
\stackrel{R}{\Lambda}\end{array}$ & 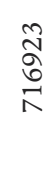 & $\begin{array}{c}\hat{n} \\
\hat{n} \\
\hat{n}\end{array}$ & $\begin{array}{l}\text { ते } \\
\stackrel{0}{0} \\
\stackrel{1}{1}\end{array}$ & $\begin{array}{l}\stackrel{n}{0} \\
\stackrel{0}{0} \\
\stackrel{1}{1}\end{array}$ & 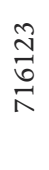 & $\begin{array}{l}\stackrel{n}{\stackrel{\sigma}{0}} \\
\stackrel{1}{1}\end{array}$ & 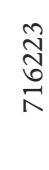 & స్ & $\begin{array}{l}\stackrel{\Re}{3} \\
\stackrel{0}{R}\end{array}$ & & $r$ & $\mathrm{r}$ & & & 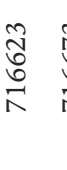 & 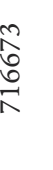 & & 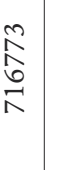 \\
\hline$\underset{\pi}{\pi}$ & $\frac{F}{\vec{I}} \frac{\exists}{\vec{J}}$ & $\exists$ & $\exists$ & $\exists$ & $\exists$ & 7 & $\exists$ & 7 & $\exists$ & $\exists$ & & 7 & 7 & & & & & & & & & $\exists$ & & & $\exists$ \\
\hline
\end{tabular}




\begin{tabular}{|c|c|c|c|c|c|c|c|c|c|c|c|c|c|c|c|c|c|c|c|c|c|c|c|c|c|c|}
\hline 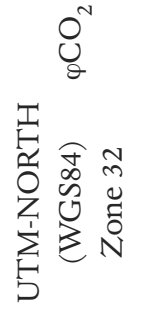 & 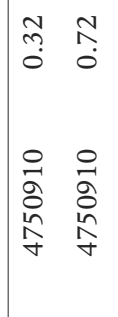 & 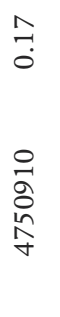 & $\begin{array}{ll}0 & 5 \\
0 & 1 \\
0 & c \\
& \\
0 & \\
0 & \\
0 & 0 \\
0 & 0 \\
h & 5 \\
f & 5\end{array}$ & $\begin{array}{l}b^{\circ} \\
h^{\circ} \\
\text { f }\end{array}$ & 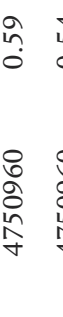 & 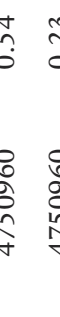 & & $\begin{array}{l}8 \\
\text { ò } \\
\text { ò } \\
\text { h }\end{array}$ & 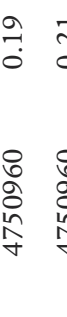 & & & 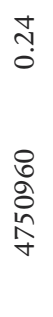 & 占 & 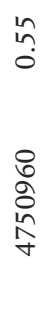 & & $\stackrel{\circ}{\stackrel{\circ}{\circ}}$ & & & & & & & & $p$ & & 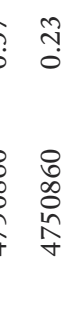 \\
\hline 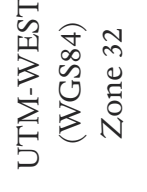 & 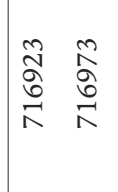 & 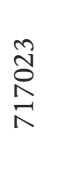 & 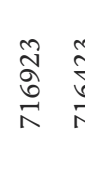 & & 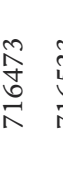 & 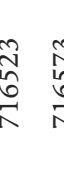 & & 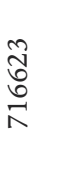 & 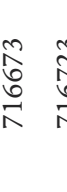 & & 趈 & 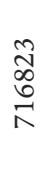 & 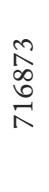 & $\begin{array}{l}\text { â } \\
\text { ถે }\end{array}$ & & 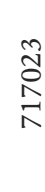 & & $\checkmark$ & & & & & & ్ֶ & & $\hat{i}_{\hat{0}}^{\infty}$ \\
\hline$\stackrel{\mathscr{\Xi}}{\Xi}$ & 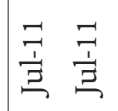 & $\vec{\Xi}$ & & & $\frac{7}{3}$ & $\frac{7}{3} \frac{7}{3}$ & & & $\frac{7}{3} \overline{3}$ & & & $\stackrel{\Xi}{\Xi}$ & $\stackrel{\Xi}{\Xi}$ & $\exists$ & & $\vec{\exists}$ & & 7 & & & & & & 7 & & $\frac{\Xi}{3}$ \\
\hline$\bigcirc_{\mathscr{Q}}^{N}$ & त̂ & $\stackrel{\text { no }}{\stackrel{r}{r}}$ & $\begin{array}{r}\hat{\lambda} \\
0 \\
0\end{array}$ & & $\begin{array}{l}0 \\
\text { bे } \\
0\end{array}$ & $\begin{array}{ll}\infty & 5 \\
\infty & 5 \\
0 & 5 \\
0 & c\end{array}$ & S & in & $\overbrace{0}^{0}$ & $\frac{5}{0}$ & 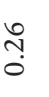 & $\stackrel{0}{\circ}$ & ถู & $\stackrel{8}{0}$ & & $\begin{array}{l}6 \\
0 \\
0\end{array}$ & & ๙ & & & & & & $\stackrel{\infty}{\sim} \underset{\sim}{\sim}$ & & \\
\hline 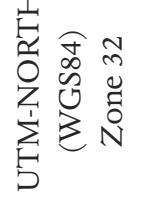 & 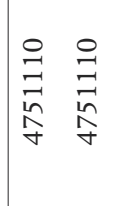 & 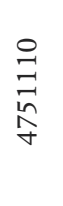 & 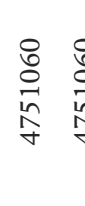 & & 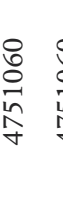 & 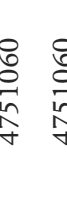 & & 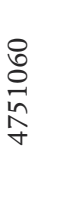 & 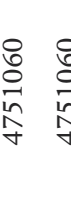 & & 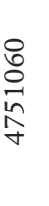 & $\begin{array}{l}\stackrel{8}{0} \\
\stackrel{1}{n} \\
\hat{f}\end{array}$ & 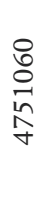 & $\begin{array}{l}\stackrel{8}{\circ} \\
\stackrel{1}{n} \\
\hat{\sigma}\end{array}$ & & $\begin{array}{l}\stackrel{8}{\circ} \\
\stackrel{1}{n} \\
\hat{f}\end{array}$ & & 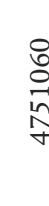 & & & & & & $\begin{array}{l}\vec{c} \\
\substack{n \\
r}\end{array}$ & & \\
\hline 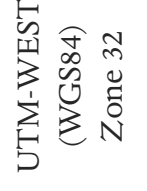 & 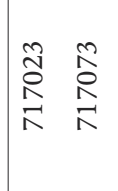 & 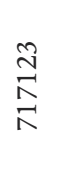 & 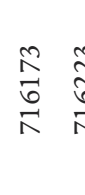 & & $\begin{array}{ll}\stackrel{N}{0} & \vdots \\
& \vdots \\
& 1\end{array}$ & 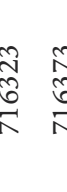 & & 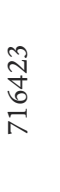 & 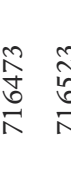 & & 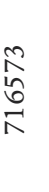 & 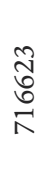 & 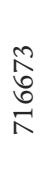 & 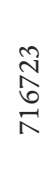 & & $\begin{array}{l}\widetilde{N} \\
\stackrel{0}{0} \\
\stackrel{\Lambda}{1}\end{array}$ & & శ్రి & & & & & & 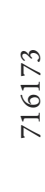 & & \\
\hline ڤัँ & 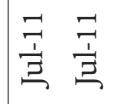 & $\vec{\Xi}$ & $\bar{\Xi} \overline{3}$ & & $\overline{3} \overline{3}$ & $\frac{7}{3} \overline{3}$ & & $\frac{\overrightarrow{1}}{\Xi}$. & $\frac{7}{\Xi} \frac{7}{3}$ & & $\bar{\Xi}$ & $\vec{\Xi}$ & $\frac{\Xi}{\Xi}$ & $\vec{\Xi}$ & & $\frac{7}{3}$ & & & & & & & & $\exists$ & & \\
\hline$\stackrel{\delta}{\sigma}_{\sigma}^{N}$ & $\begin{array}{ll}\underset{+}{+} & \vec{t} \\
-i & 0\end{array}$ & $\stackrel{\tilde{b}}{0}$ & $\stackrel{\substack{c \\
0}}{0}$ & ?ִ & ‡ै & $\begin{array}{ll}0 & 15 \\
0 & 0\end{array}$ & t. & $\begin{array}{l}\text { in } \\
\text { on } \\
0\end{array}$ & $\begin{array}{cc}\vec{m} & n \\
0 & 0\end{array}$ & ?ै? & n̂n & $\tilde{n}$ & $\underset{\substack{0 \\
0}}{0}$ & $\stackrel{ \pm}{0}$ & & $\stackrel{\infty}{1}$ & ๙ิ & ১ & & & & ñ? & & : & & \\
\hline 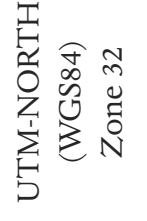 & 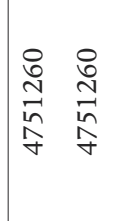 & 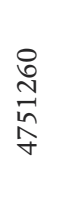 & 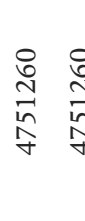 & & 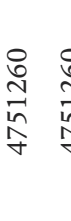 & 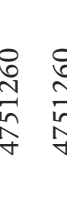 & & 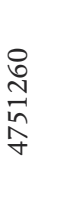 & 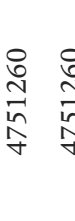 & & 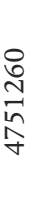 & $\begin{array}{l}\stackrel{8}{0} \\
\stackrel{1}{1} \\
\hat{n} \\
\text { f }\end{array}$ & 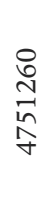 & $\begin{array}{l}\stackrel{8}{0} \\
\text { In } \\
\\
\text { f }\end{array}$ & & $\begin{array}{l}\stackrel{8}{0} \\
\stackrel{1}{n} \\
\hat{n} \\
f\end{array}$ & $\frac{0}{n}$ & \begin{tabular}{l}
$\stackrel{\circ}{N}$ \\
$\frac{1}{n}$ \\
\multirow{7}{7}{}
\end{tabular} & in & & 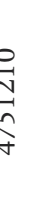 & $\stackrel{ }{\overrightarrow{1}}$ & 3 & $\begin{array}{l}\stackrel{0}{1} \\
\stackrel{\sqrt{n}}{\mathfrak{r}} \\
\stackrel{f}{\sigma}\end{array}$ & & \\
\hline 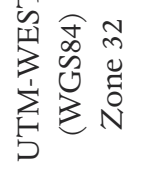 & 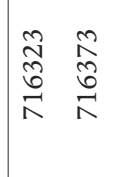 & 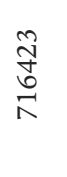 & 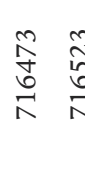 & & 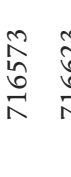 & 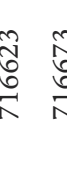 & & 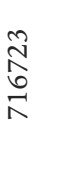 & 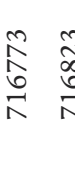 & & 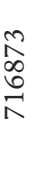 & 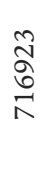 & 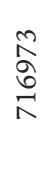 & $\begin{array}{l}\stackrel{\overbrace{}}{2} \\
\stackrel{1}{ }\end{array}$ & 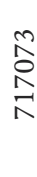 & 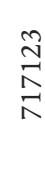 & & $\frac{\widetilde{乛}}{\mathbb{Z}}$ & 8 & & 3. & $\approx$ & 5 & 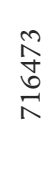 & & \\
\hline ڤัँ & 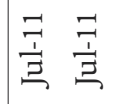 & $\overrightarrow{\bar{\Xi}}$ & $\bar{\exists} \overline{3}$ & & $\overrightarrow{7}$ & $\begin{array}{l}\overline{3} \\
\bar{E}\end{array}$ & & $\vec{\Xi}$ & $\frac{7}{3} \overline{3}$ & & $\exists$ & $\frac{7}{3}$ & $\vec{\Xi}$ & $\vec{\Xi}$ & & $\exists$ & & & & & & & & $\exists$ & & \\
\hline$\overbrace{\mathscr{\sigma}}^{N}$ & $\begin{array}{ll}\text { त̂े } & \infty \\
0 & \overrightarrow{0}\end{array}$ & กิ & $\vec{\sigma} \underset{c}{a}$ & : & 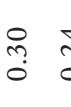 & $\begin{array}{ll}\text { İ } \\
\text { c. }\end{array}$ & t+ & 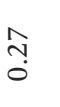 & $\begin{array}{ll}\stackrel{\infty}{1} & \text { nf } \\
0 & 0\end{array}$ & $\underline{n}$ & त̂̀ & $\stackrel{\text { in }}{0}$ & กิ & ?n & & $\stackrel{\sim}{\text { กิ }}$ & & $m$ & & & & & & $\widehat{\widehat{a}}$ & & \\
\hline 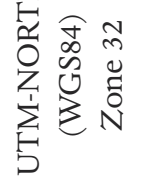 & 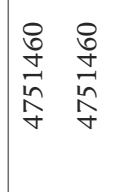 & 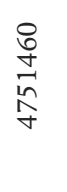 & 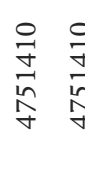 & & 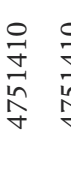 & 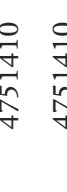 & & 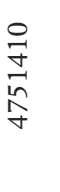 & 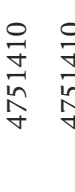 & & 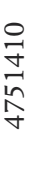 & $\begin{array}{l}\stackrel{\partial}{\vec{t}} \\
\vec{n} \\
\forall\end{array}$ & 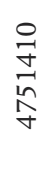 & 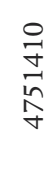 & $\stackrel{\circ}{\vec{J}}$ & 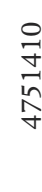 & 5 & 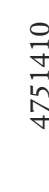 & 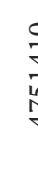 & & $\stackrel{f}{n}$ & & 7 & 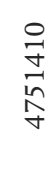 & & \\
\hline 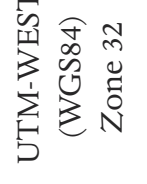 & 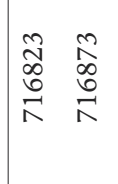 & $\begin{array}{l}\text { à } \\
\text { ò } \\
\text { 1 }\end{array}$ & 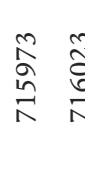 & & 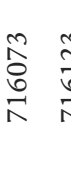 & 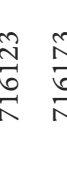 & & $\begin{array}{l}\text { त̃ } \\
\text { ָे } \\
\text { N }\end{array}$ & 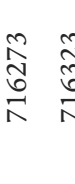 & & $\begin{array}{l}\text { ñ } \\
\hat{3} \\
\text { त } \\
\end{array}$ & $\begin{array}{l}\text { I } \\
\text { Jै } \\
\stackrel{N}{1}\end{array}$ & 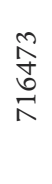 & 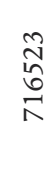 & 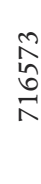 & 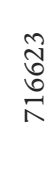 & & $\frac{\pi}{\mathbb{E}}$ & & & & & & $\hat{\tilde{b}}$ & & \\
\hline$\stackrel{\varpi}{\pi}$ & $\frac{7}{3}$ & $\frac{7}{7}$ & $I$ & & $\frac{I}{\exists}$ & $\bar{I}$ & & $\frac{1}{7}$ & $\exists$ & & & r & & 7 & & 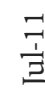 & & & & & & & & . & & \\
\hline
\end{tabular}




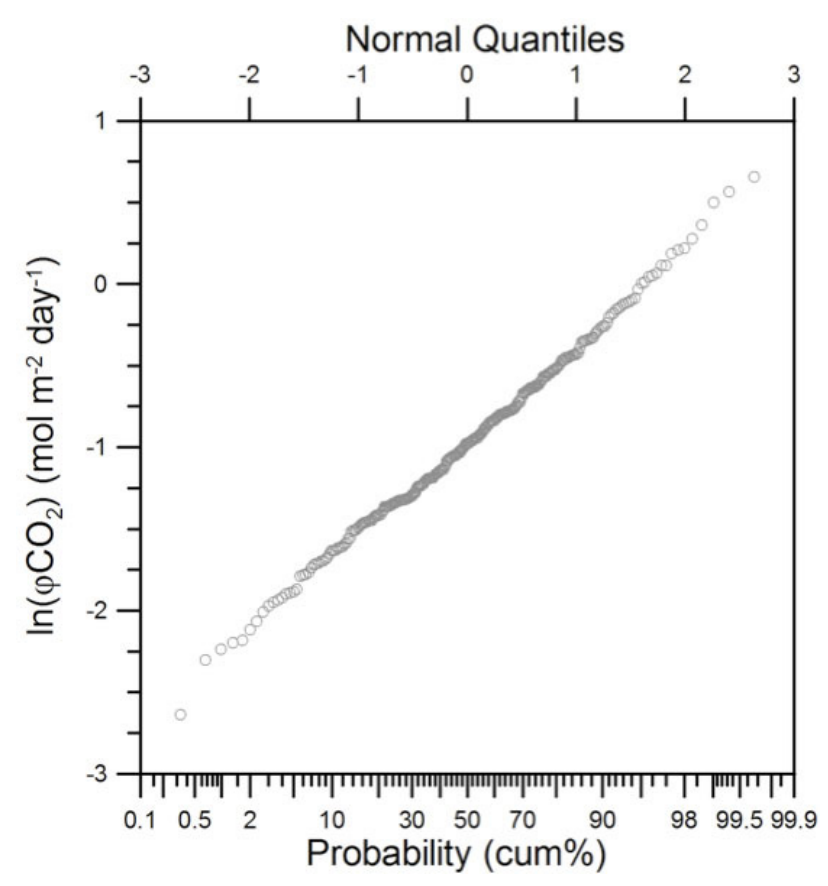

Figure 3. Probability plots of $\varphi \mathrm{CO}_{2}$ values obtained by applying the partitioning method of Sinclair [1974, 1991]. Circles show the measured soil diffuse $\varphi \mathrm{CO}_{2}$ (in $\mathrm{mol} \mathrm{m}^{-2}$ day $\left.^{-1}\right)$.

log-normal Kriging method [e.g. Krige 1951, Matheron 1970] using the ISATIS (C) software package of Geovariances. The processing of the geostatistical data indicates that the spherical model with a nugget effect is the best model to describe the spatial variability of $\varphi \mathrm{CO}_{2}$. The variogram parameters have a range of $115 \mathrm{~m}$, a sill of 0.12 and a nugget of $0.2 \varphi \mathrm{CO}_{2} \mathrm{~mol} \mathrm{~m}^{-2}$ day $^{-1}$. The iso-flux map shows that the diffuse $\varphi \mathrm{CO}_{2}$ is generally low (average value of $0.38 \mathrm{~mol} \mathrm{~m}^{-2} \mathrm{day}^{-1}$ ), although values $>0.6$ and up to $3.42 \mathrm{~mol} \mathrm{~m}^{-2}$ day $^{-1}$ were occasionally measured at NW and SW and E, respectively, of the studied area (Figure 6). Since the $\mathrm{CO}_{2}$ flux values tended to be log-normally distributed, the whole frequency distribution was analyzed by using a Gaussian (normal) distribution in the complete data set (Figure 3 ), and the $\ln \left(\varphi \mathrm{CO}_{2}\right)$ data were processed according to the method proposed by Sinclair [1974].

The Sinclair procedure allows to choose threshold values between anomalous and background data using probability graphs. On a log-probability plot, a single log-normal population results as a straight line, whereas a curve with an inflection point describes the theoretical distribution of two overlapping log-normal populations and $n$ overlapping log-normal populations result

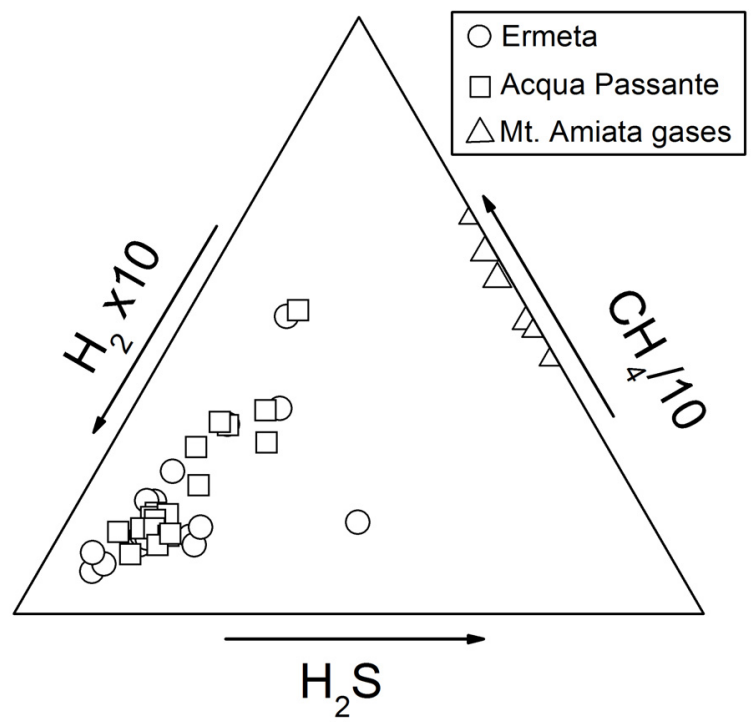

Figure 4. $\mathrm{H}_{2} \mathrm{~S}-\mathrm{CH}_{4}-\mathrm{H}_{2}$ ternary diagram for the Ermeta and Acqua Passante gases and the Mt. Amiata gas discharges (by Tassi et. al. [2009]).

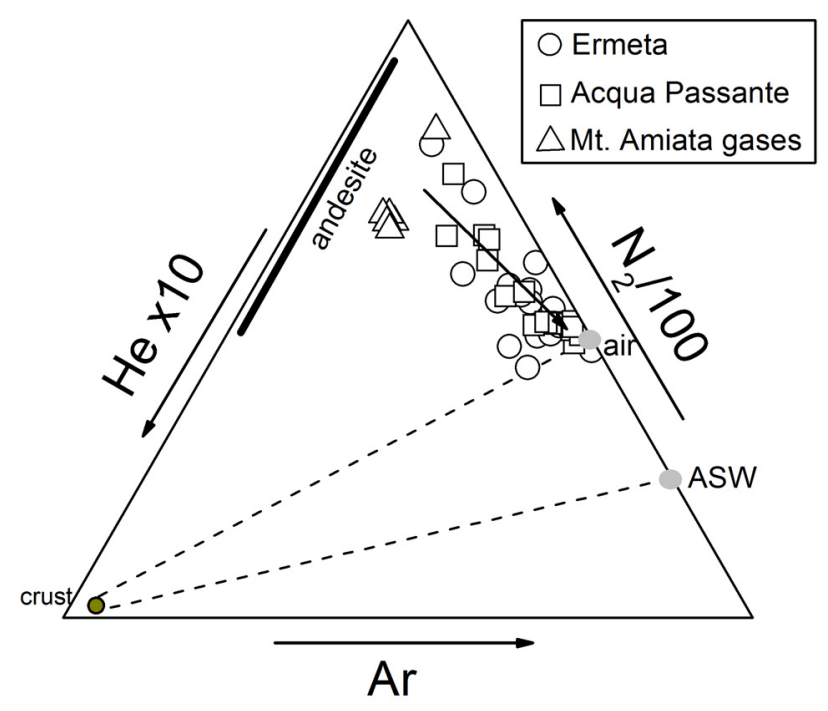

Figure 5. $\mathrm{N}_{2}-\mathrm{He}-\mathrm{Ar}$ ternary diagram for the Ermeta and Acqua Passante gases and the Mt. Amiata gas discharges (by Tassi et. al. [2009]). ASW: air-saturated-water (ASW).

on a curve characterized by $n-1$ inflection points. Generally speaking, the log-probability plot shows a straight line for the $\mathrm{CO}_{2}$ flux data related to the Ermeta area (Figure 3).

\begin{tabular}{ccccccc}
\hline $\begin{array}{c}\text { Surveyed } \\
\text { surface }\left(\mathrm{m}^{2}\right)\end{array}$ & $\begin{array}{c}\text { Number of } \\
\text { measurements }\end{array}$ & Mean & Median & Minimum & Maximum & Std. Dev. \\
\hline 653,550 & 301 & -0.95 & -0.97 & -2.63 & 1.23 & 0.56 \\
\hline
\end{tabular}

Table 3. Surveyed surface (in $\mathrm{m}^{2}$ ), number of measurements and statistical parameters (mean, median, minimum, maximum and standard deviation) of the $\varphi \mathrm{CO}_{2}$, expressed as $\ln \left(\varphi \mathrm{CO}_{2}\right)\left(\mathrm{mol} \mathrm{m}^{-2}\right.$ day $\left.^{-1}\right)$. 


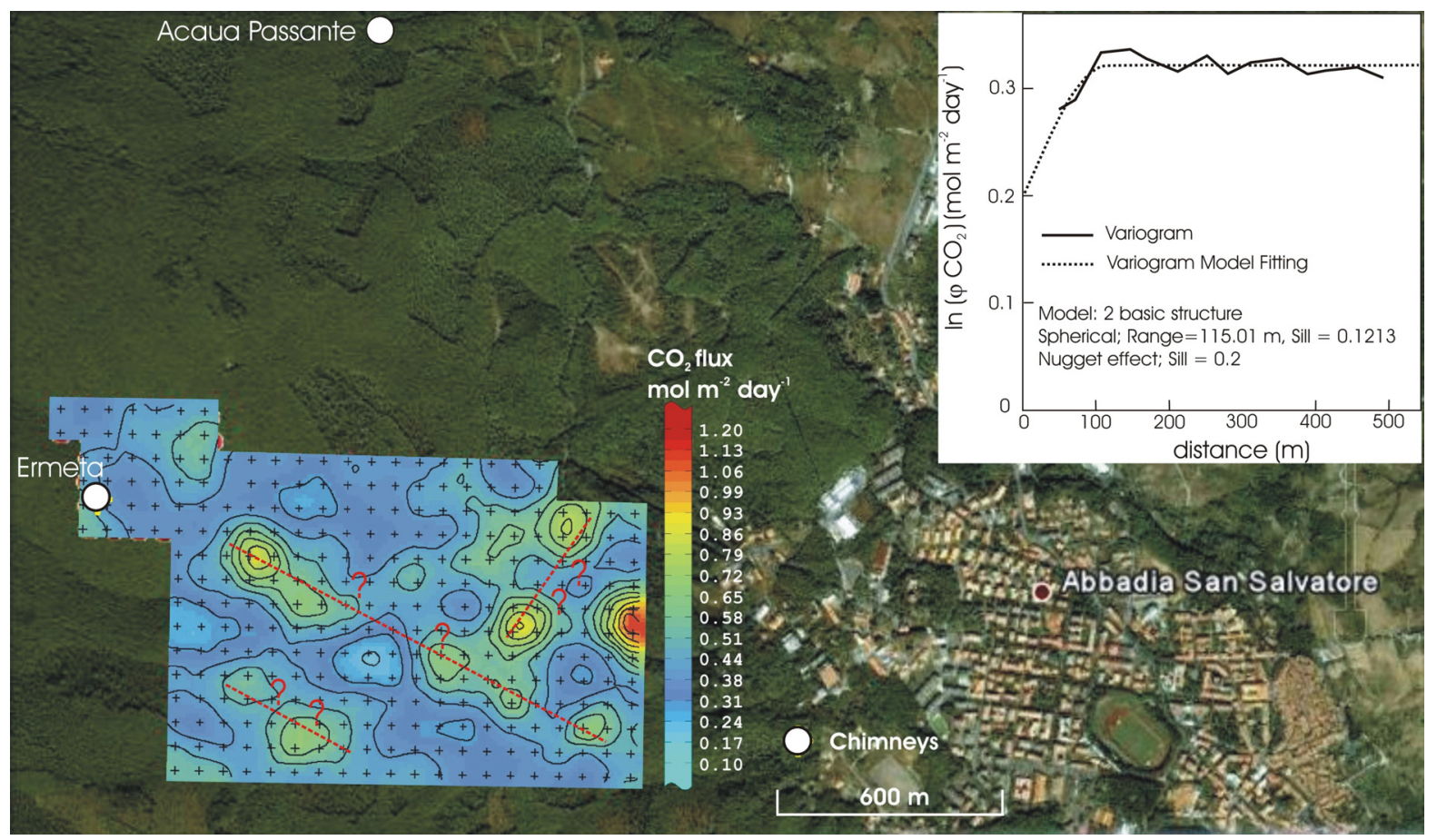

Figure 6. Map of soil diffuse $\varphi \mathrm{CO}_{2}$ (in mol m${ }^{-2}$ day $^{-1}$ ) for the area close to the Ermeta well. Red dashed lines refer to preferential alignments (faults or fractures) where highest $\mathrm{CO}_{2}$ flux values were measured.

On the basis of this reasonable consideration, the data set can be modeled with a population that can be considered as the "local $\mathrm{CO}_{2}$ flux" (average value of $0.38 \mathrm{~mol} \mathrm{~m}^{-2}$ day $\left.^{-1}\right)$ and comparable with the mean biological $\mathrm{CO}_{2}$ flux $\left(\sim 0.11 \mathrm{~mol} \mathrm{~m}^{-2}\right.$ day ${ }^{-1}$; e.g. Raich and Schleisinger [1992]). The respective statistical parameters of the considered population are reported in Table 4.

The total $\mathrm{CO}_{2}$ output for the Ermeta area was calculated by applying the Sichel's t-estimator $\left(M_{\mathrm{i}}\right)$ [David 1977], whose value was derived by multiplying $M_{i}$ times the area covered by the population (Table 4). In the same way, the central $95 \%$ confidence intervals of the total $\mathrm{CO}_{2}$ output were used to calculate the uncertainty of the population. According to the Sichel's $t$-estimator $\left(M_{\mathrm{i}}\right)$, the total amount of $\mathrm{CO}_{2}$ released through diffuse degassing is estimated to be 11.81 ton day $^{-1}$.

5.3. Computation of the amount of dangerous gases released from the Ermeta and Acqua Passante wells since their construction and their relationships with the soil diffuse gases

The impact of the hydrothermal gases released to the atmosphere from the two chimneys is of critical im- portance for the municipality of Abbadia San Salvatore since disagreeable effects for people approaching these areas, such as odor of rotten eggs, are clearly felt. Furthermore, locally lethal gas accumulation in air may occur as testified by the presence, though rare, of dead animals found in proximity of the wells.

Our data show that the chemical composition of the well gases in the last 5 years had no significant variations and the $\mathrm{CO}_{2}$ flow rate from the two chimneys has remained fairly constant during the period of observation. Consequently, we may reasonably assume that the amount of $\mathrm{CO}_{2}$ has not significantly changed since the construction of the Ermeta and Acqua Passante wells, i.e. 54 and 75 years ago, respectively. This allows us to compute that since then the two wells have emitted up to 850,000 tons of $\mathrm{CO}_{2}$ in the atmosphere. This calculation can be extended to $\mathrm{H}_{2} \mathrm{~S}$ and $\mathrm{CH}_{4}$ on the basis of the measured mean values of the $\mathrm{H}_{2} \mathrm{~S} / \mathrm{CO}_{2}$ $($ Ermeta $=0.0005$ and Acquapassante $=0.0004)$ and $\mathrm{CH}_{4} / \mathrm{CO}_{2}$ (Ermeta $=0.0063$ and Acquapassante $=$ 0.0056) ratios, obtained by the 5 years of periodic sampling collection of the well gases. Thus, the total amount of $\mathrm{H}_{2} \mathrm{~S}$ and $\mathrm{CH}_{4}$ emitted from the two chim-

\begin{tabular}{cccccc}
\hline Population & $\begin{array}{c}\text { Number of } \\
\text { measuraments }\end{array}$ & $\mathrm{CO}_{2}$ flux population & $\begin{array}{c}\text { mean } \ln \left(\varphi \mathrm{CO}_{2}\right) \\
\text { flux value }\left(\mathrm{M}_{\mathrm{i}}\right)\end{array}$ & $\begin{array}{c}\text { total diffuse } \\
\mathrm{CO}_{2} \text { output } \\
\text { (ton/day) }\end{array}$ & $\begin{array}{c}95 \% \\
\text { confidence interval } \\
\text { (ton/day) }\end{array}$ \\
\hline 1 & 301 & local background & -1.076 & 11.81 & $13.07-10.84$ \\
\hline
\end{tabular}

Table 4. Estimated parameters of partitioned populations and derived total $\mathrm{CO}_{2}$ output calculated according to Sinclair [1974] and Sichel's $t$-estimator $\left(M_{\mathrm{i}}\right)[$ David 1977]. 
neys is of about 430 and 5,150 tons, respectively, the Ermeta well discharging slightly less than one order of magnitude the amount of $\mathrm{H}_{2} \mathrm{~S}$ and $\mathrm{CH}_{4}$ released by that of Acqua Passante.

It is worth noting that the sum of $\mathrm{CO}_{2}$ released on a year basis from Ermeta and Acqua Passante wells was up to twice the amount of $\mathrm{CO}_{2}$ emitted from bubbling pools and dry vents computed in the surrounding areas of Mt. Amiata, such as: Acqua Borra at Castelnuovo Berardenga (Siena) ( 5 ton $\mathrm{y}^{-1}$; Vaselli et al., unpublished data), Lo Spuntone, Il Palazzo and Buca alle Colline at Campiglia d'Orcia (Siena) $\left(\approx 370\right.$ ton $\mathrm{y}^{-1}$ at each site; Vaselli et al. [2006a], Tassi et al. [2009]), Bossoleto at Rapolano (Siena) (4,380 ton $\mathrm{y}^{-1}$; Van Gardingen et al. [1995], Vaselli et al. unpublished data), Pienza (5,475 ton $\mathrm{y}^{-1}$; Giuli et al. [1997]) and Ambra River (7,300 ton/year; Cuccoli et al. [2006]). A similar comparison was not computed for $\mathrm{H}_{2} \mathrm{~S}$ and $\mathrm{CH}_{4}$ since in most cases the $\mathrm{CO}_{2}$ flux was determined by open-path IR laser measurements [Belotti et al. 2003], which were related to the gas discharges from various vents, contributing to the calculated amount of $\mathrm{CO}_{2}$. No specific investigations were performed to highlight possible differences in the $\mathrm{H}_{2} \mathrm{~S} / \mathrm{CH}_{4}$ ratios from each emission discharging from a certain site. If present, the calculated amount of $\mathrm{H}_{2} \mathrm{~S}$ and $\mathrm{CH}_{4}$ might be affected by a large error.

The generally low $\mathrm{CO}_{2}$ soil flux values measured in SE the Ermeta well seem to indicate that the diffuse gas emission from the soil is mainly related to soil respiration. Plant roots and soil microbial activities are expected to produce a relatively homogeneous distribution of the diffuse soil $\mathrm{CO}_{2}$ when a soil cover is present, likely modulated by seasonal patterns of moisture availability, land use and biological cycles [e.g. Almagro et al. 2009, Elío et al. 2013 and references therein]. As previously mentioned, the studied area is generally characterized by a well-developed vegetation and no measurements were performed where the volcanic rocks were outcropping. Nevertheless, the spatial distribution of the $\mathrm{CO}_{2}$ fluxes (Figure 6) shows the occurrence of preferential NW-SE and NNE-SSW oriented alignments characterized by relatively high $\mathrm{CO}_{2}$ flux values, which might be related to weakness zones, such as fractures and/or faults. As a first approximation, these sites may suggest the presence of gases fed by the deep-seated reservoir. In this respect, an isotopic survey of the $\mathrm{CO}_{2}$ soil gases is required to assess whether these zones can be regarded as the preferential pathways for the uprising of deep-originated $\mathrm{CO}_{2}$. The carbon isotopic signature of the Ermeta gas ( $-3.5 \mathrm{~V}-\mathrm{PDB} \%$ ), as well as that of Acqua Passante, is indeed significantly different with respect to that associated with a biogenic source ( $\sim 20$ V-PDB \%; Schoell [1980]). To further support this hypothesis a geostructural survey is required to verify the correspondence between the geochemical and geological signals.

\subsection{Is the closure of the Ermeta and Acqua Passante} wells feasible and safe?

Since 2008, the municipality of Abbadia San Salvatore has become the owner of the mining concession from where cinnabar was extracted and in agreement with the authorities of the Tuscany Region it was appointed to i) monitor the gases discharged from the Ermeta and Acqua Passante wells and ii) actuate prevention measurements to minimize such emissions to the atmosphere. As previously mentioned, the two wells are located in the proximity of tourist areas and the reduction of their gas discharges is expected to provide benefits in terms of safety and healthy conditions.

Presently, $\sim 15,000$ tons of $\mathrm{CO}_{2}$, along with 8 and 92 tons of $\mathrm{H}_{2} \mathrm{~S}$ and $\mathrm{CH}_{4}$, respectively, are emitted on a yearly basis from the Ermeta and Acqua Passante chimneys and they are related to the discharge of a natural subsurface $\mathrm{CO}_{2}$ reservoir intercepted during $\mathrm{Hg}$ prospection campaigns few kms away from Abbadia San Salvatore. The Ermeta well contributes for $>85 \%$ of the total $\mathrm{CO}_{2}$ discharge. The amount of $\mathrm{CO}_{2}$ is more than three times higher than that released by diffuse soil degassing released from an area of $653,550 \mathrm{~m}^{2}$ located SE of the Ermeta chimney, this value being of 4,310 ton $\mathrm{y}^{-1}$.

According to the aforementioned agreement, the municipality of Abbadia San Salvatore would intend to proceed with the closure of one of the wells and that of Ermeta was thought to be considered as the most suitable since it is i) discharging a larger quantity of gas, ii) located more far away from the main roads and iii) surrounded by a vegetation (and soil) cover (Figure 2), similar to that where $\varphi \mathrm{CO}_{2}$ fluxes were directly measured suggesting that the diffuse soil $\mathrm{CO}_{2}$ can be assumed to be only affected by soil respiration, e.g. no gas discharges are present at the surface.

Nevertheless, one of the most critical points is to understand and recognize which are those sites that might possibly be affected by potential leakage or micro-seepage of $\mathrm{CO}_{2}$ [e.g. Klusman 2013, Elío et al. 2013, Nisi et al. 2013 and references therein] as gases in deep reservoirs are buoyant and tend to vertically or laterally migrate following the most permeable pathways in response to pressure. Several authors have proposed buoyancy of gas (micro)bubbles [e.g. MacElvain 1969, Davidson 1982, Klusman 1993, Klusman and Saeed 1996] as a potential process of vertical migrations of gases and gas overpressuring underground, as that expected once the closure of the Ermeta well will occur, plays an important role [Klusman 1997]. In this respect, 
faults and fractures, may then vehiculate the $\mathrm{CO}_{2}$-rich gases upwards [e.g. Etiope 1999, Brown 2000 and references therein]. As pinpointed by Klusman [2013], the rapidity of transport is another important parameter to be taken into account.

It appears to be realistic the fact that the weakness zones, individuated by the direct measurements of the $\mathrm{CO}_{2}$ soil fluxes (Figure 6), can likely be affected by an increase in the $\mathrm{CO}_{2}$ flux, being these areas characterized by a higher permeability. This would theoretically imply the possibility to recognize those areas from where a higher probability of $\mathrm{CO}_{2}$ leakage is expected in the case of a gas overpressure by applying a relatively simple, though effective, method such as that of the accumulation chamber. Owing to the lack of specific investigations addressed to a detailed geostructural study in the surrounding areas of the Ermeta well, to tentatively reduce the discharge of $\mathrm{CO}_{4}, \mathrm{CH}_{4}$ and $\mathrm{H}_{2} \mathrm{~S}$ we highly recommend the municipality of Abbadia San Salvatore to solely temporarily close the Ermeta well by using $\mathrm{N}_{2}$-filled air-bags before a definitive cementation. Although it is difficult to predict the side effects deriving by such an action, we may speculate that an increasing gas flow rate could be expected. Furthermore, no indications are also presently available about the timing before a modification of the soil diffuse $\mathrm{CO}_{2}$ is to be recorded. This implies that a geochemical and isotopic monitoring program has to be designed and mainly focused on the diffuse soil $\mathrm{CO}_{2}$ with particularly reference to the recognized weakness zones. In the case of increasing activity in terms of release of soil $\mathrm{CO}_{2}$, the tube closing being temporary, previous conditions could easily be restored by re-opening the Ermeta well. Thus, currently there are no sufficient clues to definitively shut down the Ermeta well. A similar approach should be considered if the closure of the Acqua Passante well is to be considered.

\section{Conclusions}

The municipality of Abbadia San Salvatore is intending to reduce the impact related to the emission in the atmosphere of $\mathrm{CO}_{2}\left(\mathrm{H}_{2} \mathrm{~S}, \mathrm{CH}_{4}\right)$-rich gases by two wells: Acqua Passante and Ermeta, built in 1938 and 1959, respectively, during $\mathrm{Hg}$ prospection campaigns and located in the eastern part of the Mt. Amiata volcanic system. The easiest solution to avoid the gas discharge is to close down the wells, the Ermeta drilling being the one to be tested first.

In this work we have determined that the Ermeta and Acqua Passante drillings are emitting up to 15,000 ton $\mathrm{y}^{-1}$ of $\mathrm{CO}_{2}$ and 92 and 8 ton $\mathrm{y}^{-1}$ of $\mathrm{CH}_{4}$ and $\mathrm{H}_{2} \mathrm{~S}$, respectively. These gases can be regarded as the discharge of a natural subsurface $\mathrm{CO}_{2}$-rich reservoir. The origin of these gases is similar to those naturally released from dry vents and bubbling pools located few kilometers away from the two wells, with the exception of $\mathrm{H}_{2}$, whose higher content is apparently related to corrosion processes of the iron tubing of the chimneys as the deep-seated gases flow through them. We have also estimated the $\mathrm{CO}_{2}$ output from soil $\left(4,310\right.$ ton $\mathrm{y}^{-1}$ in an area of about $653,500 \mathrm{~m}^{2}$ ) close to the Ermeta well and determined by the accumulation chamber method. Relatively high $\mathrm{CO}_{2}$ fluxes were recognized along preferential alignments, possibly associated with the presence of faults or fractures (Figure 6).

Standing the absence of detailed geostructural investigations nearby Ermeta, we strongly recommend a temporarily closure of the Ermeta well by means of $\mathrm{N}_{2}$-filled air-bags before proceeding with a definitive cementation. Afterwards, an appropriate geochemical and isotopic monitoring activity of the soil gas is required to verify the presence of leakage and microseepage of $\mathrm{CO}_{2}$.

Acknowledgements. Many thanks are due to the Municipality of Abbadia San Salvatore that partly financially the present study. The Authors are grateful to Tom Darrah for providing the helium isotopic ratios of the Ermeta and Acqua Passante gases. The personnel of West System is thanked for their help in the installation of the hotwire anemometer in the Acqua Passante and Ermeta wells. F. Frondini and two anonymous reviewers are kindly acknowledged for their suggestions and comments to an early version of the manuscript.

\section{References}

Allard, P., J. Carbonnelle, D. Dajleric, J. Le Bronec, P. Morel, M.C. Robe, J.M. Maurenas, R. Faivre-Pierret, D. Martin, J.C. Sabroux and P. Zettwoog (1991). Eruptive and diffusive emissions of $\mathrm{CO}_{2}$ from Mt. Etna, Nature, 351, 387-391.

Almagro, M., J. López, I. Querejeta and M. MartinezMena (2009). Temperature dependence of soil $\mathrm{CO}_{2}$ efflux is strongly modulated by seasonal patterns of moisture availability in a Mediterranean ecosystem, Soil Biol. Biochem., 41, 594-605.

Bartole, R. (1995). The North-Tyrrhenian-Northern Apennines post-collisional system: constraints for a geodynamic model, Terra Nova, 1, 7-30.

Baubron, J.C., P. Allard and J.P. Toutain (1990). Diffuse volcanic emissions of carbon dioxide from Vulcano Island, Italy, Nature, 344, 51-53.

Baubron, J.C., P. Allard and J.P. Toutain (1991). Gas hazard on Vulcano Island, Nature, 350, 26-27.

Belotti, C., F. Cuccoli, L. Facheris and O. Vaselli (2003). An application of tomographic reconstruction of atmospheric $\mathrm{CO}_{2}$ over a volcanic site based on openpath IR Laser measurement, IEEE Transact. Geosci. Rem. Sensing, 41, 2629-2637.

Brogi, A., D. Liotta, M. Meccheri and L. Fabbrini (2010). 
Transtensional shear zones controlling volcanic eruptions: the Middle Pleistocene Mt Amiata volcano (inner Northern Apennines, Italy), Terra Nova, 22, 137-146.

Brombach, T., J.C. Hunziker, G. Chiodini, C. Cardellini and L. Marini (2001). Soil diffuse degassing and thermal energy fluxes from the southern Lakki plain, Nisyros (Greece). Geophys. Res. Lett., 28, 69-72.

Brown, A. (2000). Evaluation of possible gas microseepage mechanisms, Am. Assoc. Petrol. Geol. Bull., 84, 1775-1789.

Burton, M.R., G.M. Sawyer and D. Granieri (2013). Deep Carbon Emissions from Volcanoes, Rev. Min. Geochem., 75, 323-354.

Cadoux, A., and D.L. Pinti (2009). Hybrid character and pre-eruptive events of Mt. Amiata volcano (Italy) inferred from geochronological, petro-geochemical and isotopic data, J. Volcanol. Geotherm. Res., 179, 169-190.

Calamai, A., R. Cataldi, P. Squarci and L. Taffi (1970). Geology, Geophysics and Hydrogeology of the Monte Amiata Geothermal Field, Geothermics Special Issue, 1, 1-9.

Carapezza, M., B. Badalamenti, L. Cavarra and A. Scalzo (2003). Gas hazard assessment in a densely inhabited area of Colli Albani Volcano (Cava dei Selci, Roma), J. Volcanol. Geotherm. Res., 123, 81-94.

Chiodini, G., F., Frondini and B. Raco (1996). Diffuse emission of $\mathrm{CO}_{2}$ from the Fossa crater, Vulcano Island (Italy), Bull. Volcanol., 58, 41-50.

Chiodini, G., R. Cioni, M. Guidi, L. Marini and B. Raco (1998). Soil $\mathrm{CO}_{2}$ flux measurements in volcanic and geothermal areas, Appl. Geochem., 13, 543-552.

Chiodini, G., F. Frondini, D.M. Kerrick, J. Rogie, F. Parello, L. Peruzzi and A.R. Zanzari (1999). Quantification of deep $\mathrm{CO}_{2}$ fluxes from Central Italy. Examples of carbon balance for regional aquifers and of soil diffuse degassing, Chem. Geol., 159, 205-222.

Chiodini, G., F. Frondini, C. Cardellini, F. Parello and L. Peruzzi (2000). Rate of diffuse carbon dioxide Earth degassing estimated from carbon balance of regional aquifers: the case of Central Apennine (Italy), J. Geophys. Res., 105, 8423-8434.

Chiodini, G., M. Todesco, S. Caliro, C. Del Gaudio, G. Macedonio and M. Russo (2003). Magma degassing as a trigger of bradyseismic events: the case of Phlegrean Fields (Italy), Geophys. Res. Lett., 30 (8), 1434; doi:10.1029/2002GL016790.

Chiodini, G., C. Cardellini, A. Amato, E. Boschi, S. Caliro, F. Frondini and G. Ventura (2004a). Carbon dioxide Earth degassing and seismogenesis in central and southern Italy, Geophys. Res. Lett., L07615; doi:10.1029/2004GL019480.
Chiodini, G., R. Avino, T. Brombach, S. Caliro, C. Cardellini, S. De Vita, F. Frondini, E. Marotta and G. Ventura (2004b). Fumarolic degassing west of Mount Epomeo, Ischia (Italy), J. Volcanol. Geotherm. Res., 133, 291-309.

Chiodini, G., D. Granieri, R. Avino, S. Caliro and A. Costa (2005). Carbon dioxide diffuse degassing and estimation of heat release from volcanic and hydrothermal systems, J. Geophys. Res., 110, , B08204; http:/ / dx.doi.org/10.1029/2004JB003541.

Chiodini, G., D. Granieri, R. Avino, S. Caliro, A. Costa, C. Minopoli and G. Vilardo (2010). Non volcanic $\mathrm{CO}_{2}$ Earth degassing: Case of Mefite d'Ansanto (southern Apennines), Italy, Geophys. Res. Lett., 37, L11303; doi:10.1029/2010GL042858.

Craig, H., and J.E. Lupton (1976). Primordial neon, helium and hydrogen in oceanic basalts, Earth Planet. Sci. Lett., 31, 369-385.

Cuccoli, F., O. Vaselli and B. Nisi (2006). Apparati laser per il telerilevamento atmosferico della $\mathrm{CO}_{2}$ e camera di accumulo per la stima del flusso di $\mathrm{CO}_{2}$ dal letto del Torrente Ambra, Internal report CNITMESSER ITALIA, Firenze (in Italian).

David, M. (1977). Geostatistical Ore Reserve Estimation, Elsevier Sci., New York, 364 pp.

Davidson, M.J. (1982). Toward a general theory of vertical migration, Oil Gas J., 80, 288-300.

Elío, J., B. Nisi, M.F. Ortega, L.F. Mazadiego, O. Vaselli and F. Grandía (2013). $\mathrm{CO}_{2}$ soil flux baseline at the $\mathrm{CO}_{2}$ Injection pilot Plant of Hontomin (Burgos, Spain), Intl. J. Green. Gas Control., 18, 224-236.

Etiope, G. (1999). Subsoil $\mathrm{CO}_{2}$ and $\mathrm{CH}_{4}$ and their advective transfer from faulted grassland to the atmosphere, J. Geophys. Res., 104D, 889-894.

Ferrari, L., S. Conticelli, L. Burlamacchi and P. Manetti (1996). Volcanological evolution of the Monte Amiata, Southern Tuscany: new geological and petrochemical data, Acta Vulcanol., 8, 41-56.

Frondini, F., S. Caliro, C. Cardellini, G. Chiodini and N. Morgantini (2009). Carbon dioxide degassing and thermal Energy relese in the Monte Amiata volcanic-geothermal area (Italy), Appl. Geochem., 24, 860-875.

Gambardella, B., C. Cardellini, G. Chiodini, F. Frondini, L. Marini, G. Ottonello and M. Vetuschi Zoccolino (2004). Fluxes of deep $\mathrm{CO}_{2}$ in the volcanic areas of central- southern Italy, J. Volcanol. Geotherm. Res., 136, 31-52.

Gerlach, T.M. (1991). Present-day $\mathrm{CO}_{2}$ emissions from Volcanoes, EOS, 72, 249-255.

Gianelli, G., M. Puxeddu, F. Batini, G. Bertini, I. Dini, E. Pandeli and R. Nicolich (1988). Geological model of a young volcano-plutonic system: the geothermal 
region of Monte Amiata (Tuscany, Italy), Geothermics, 17, 719-734.

Gibbins, J., and H. Chalmers (2008). Carbon capture and storage, Energy Policy, 36, 4317-4322.

Giuli, D., L. Facheris, S. Tanelli and F. Cuccoli (1997). Studio preliminare di un sistema tomografico all'infrarosso per la rilevazione della distribuzione di componenti atmosferici mediante satellite, Technical Report, University of Florence, Department of Electronic Engineering, (In Italian).

Hoefs, J. (1997). Stable Isotope Geochemistry, 4th ed., Springer, Verlag, Berlin, Germany, 201 pp.

Kanemasu, E.T., W.L. Power and J.W. Sij (1974). Field chamber measurements of $\mathrm{CO}_{2}$ flux from soil surface, Soil Sciences, 118 (4), 233-237.

Klemm, D.D., and N. Neumann (1984). Ore-controlling factors in the $\mathrm{Hg}-\mathrm{Sb}$ province of southern Tuscany, Italy, In: A. Wauschkuhn et al. (eds.), Syngenesis and Epigenesis in the Formation of Mineral Deposits, Springer-Verlag, Berlin Heidelberg, 482-503.

Klusman, R.W. (1993). Soil Gas and Related Methods in Natural Resource Exploration, Wiley, Chichester, UK, 483 pp.

Klusman, R.W., and M.A. Saeed (1996). A comparison of light hydrocarbon microseepage mechanisms, In: D Schumacher and M.A Abrams (eds.), Hydrocarbon Migration and its Near-Surface Expression, Am. Assoc. Petrol. Geol., Memoir 66, 157-168.

Klusman, R.W. (1997). Modeling of microseepage of methane and light alkanes in the sedimentary column, Final Report to W.L. Gore \& Associates.

Klusman, R.W. (2013). Rate measurements and detection of gas microseepage to the atmosphere from an enhanced oil recovery/sequestration project, Rangely, Colorado, USA, Appl. Geochem., 18, 1825-1838.

Krige, D.G. (1951). A statistical approach to some basic mine valuation problems on the Witwatersrand, J. Chem. Metall. Min. Soc. S. Afr., 52, 119-139.

MacElvain, R. (1969). Mechanics of gaseous ascension through a sedimentary column, In: W.B Heroy (ed.), Unconventional Methods in Exploration for Petroleum and Natural Gas, Southern Methodist University, Dallas, TX, 15-28.

Mamyrin, B.A., and I.N. Tolstikhin (1984). Helium isotopes in nature, In: W.S. Fyfe (ed.), Development in geochemistry, Elsevier, Amsterdam, 274 pp.

Martelli, M.P., M. Nuccio, F.M. Stuart, R. Burgess, RM. Ellam and F. Italiano (2004). Helium-strontium isotope constraints on mantle evolution beneath the Roman comagmatic province, Italy. Earth. Planet. Sci. Lett., 224, 295-308.

Matheron, G. (1970). The theory of regionalized variables and its applications, Fascicule n. 5, Les Cahiers du Centre De Morphologie Mathématique, École des Mines de Paris, Fontainebleau, 211 pp.

Mauffret, A., I. Contrucci and C. Brunet (1999). Structural evolution of Northern Tyrrhenian Sea from new seismic data, Mar. Petrol. Geol., 16, 381-407.

Minissale, A., W.C. Evans, G. Magro and O. Vaselli (1997a). Multiple source components in gas manifestations from north-central Italy, Chem. Geol., 142, 175-192.

Minissale, A., G. Magro, O. Vaselli, C. Verrucchi and I. Perticone (1997b). Geochemistry of water and gas discharges from the Mt. Amiata silicic complex and surrounding areas (central Italy), J. Vulcanol. Geotherm. Res., 79, 223-251.

Minissale, A. (2004). Origin, transport and discharge of $\mathrm{CO}_{2}$ in central Italy, Earth Sci. Rev., 66, 89-141.

Montegrossi, G., F. Tassi, O. Vaselli, A. Buccianti and K. Garofalo (2001). Sulphur species in volcanic gases, Anal. Chem., 73 (3), 709-3715.

Moretti, S., O. Vaselli, B. Nisi, F. Tassi, F. Cuccoli, L. Lombardi, A. Buccianti, S. Segoni, L. Capannesi, L. Giannini, G. Montegrossi and B. Veneri (2007). Conferimento di incarico per ricerca in relazione ad alcuni aspetti legati all'analisi ed al monitoraggio di emissioni di biogas per la Direzione Rifiuti. Relazione finale, Convenzione Provincia di Firenze e Dipartimento di Scienze della Terra di Firenze, 56 pp.

Nisi, B., O. Vaselli, F. Tassi, J. Elío, A. Delgado Huertas, L.F. Mazadiego and M.F. Ortega (2013). Hydrogeochemistry of surface and underground waters in the Hontomin-Huermeces area (Burgos, Spain), Intl. J. Green. Gas Control., 14, 151-168.

Ohmoto, H., and M.B. Goldhaber (1997). Sulfur and carbon isotopes, In: H.L. Barnes (ed.), Geochemistry of Hydrothermal Ore Deposits, John Wiley and Sons, 517-611.

Pancioli, V., B. Nisi, F. Capecchiacci, O. Vaselli, F. Tassi and S. Moretti (2009). A new approach for evaluating $\mathrm{CO}_{2}$-rich gas discharges as geosites: the Acquabolle (Florence, Italy) case study, GeoActa, 8, 49-61.

Parkinson, K.J. (1981). An improved method for measuring soil respiration in the field, J. Appl. Ecol., 18, 221-228.

Peccerillo, A. (2003). Plio-Quaternary magmatism in Italy, Episodes, 26 (3), 222-226.

Peccerillo, A. (2005). Plio-Quaternary volcanism in Italy, petrology, geochemistry, geodynamics, Springer, Heidelberg, Germany.

Peccerillo, A., and M. Lustrino (2005). Compositional variations of the Plio-Quaternary magmatism in the circum-Tyrrhenian area: deep- vs. shallow-mantle processes, In: G.R. Foulger, J.H. Nathland, D.C. Pres- 
nall and D.L. Anderson (eds.), Plates, Plumes and Paradigms, Geological Society of America Special Publication, 388, 421-434.

Poreda, R.J., and K.A. Farley (1992). Rare-gases in Samoan xenoliths, Earth. Planet. Sci. Lett., 113, 129-144.

Raco, B., R. Cioni, M. Guidi, A. Scozzari, M. Lelli and G. Lippo (2006). Monitoraggio del flusso di biogas diffuso dal suolo da discariche RSU: il caso di Legoli, Peccioli (PI), Rifiuti Solidi RS, CIPA Editore, 20, 120-136.

Raco, B., M Lelli, M. Doveri, R. Cioni, M. Guidi and A. Scozzari (2008). Presentazione di una metodologia per il monitoraggio del flusso diffuso di biogas dal suolo delle discariche, Giorn. Geol. Appl., 9, 49-59.

Raich, J.W., and W.H. Schlesinger (1992). The global carbon Dioxide flux in soil respiration and its relationship to vegetation and climate, Tellus, 44B, 81-99.

Rimondi, V., J.E. Gray, P. Costagliola, O.Vaselli and P. Lattanzi (2011). Concentration, distribution, and translocation of mercury and methylmercury in mine-waste, sediment, soil, water, and fish collected near the Abbadia San Salvatore mercury mine, Monte Amiata district, Italy, Sci. Tot. Environ., 414, 318-327.

Rogie, J.D., D.M. Kerrick, M.L. Sorey, G. Chiodini and D.L. Galloway (2001). Dynamics of carbon dioxide emission at Mammoth Mountain, California, Earth Planet. Sci. Lett., 188, 535-541.

Rollinson, H. (1993). Using geochemical data. Longman, London, UK, $352 \mathrm{pp}$.

Sano, Y., and H. Wakita (1988). Helium isotope ratio and heat discharge rate in Hokkaido Island, northeastern Japan, Geochem. J., 22, 293-303.

Schoell, M. (1980).The hydrogen and carbon isotopic composition of methane from natural gases of various origins, Geochim. Cosmochim. Acta, 44, 649-661.

Sinclair, A.J. (1974). Selection of threshold values in geochemical data using probability graphs, J. Geochem. Explor., 3, 129-149.

Sinclair, A.J. (1991). A fundamental approach to threshold estimation in exploration geochemistry: probability plots revisited, J. Geochem. Expl., 41, 1-22.

Tanelli, G. (1983). Mineralizzazioni metallifere e minerogenesi della Toscana, Mem. Soc. Geol. Ital., 25, 91-109.

Tassi, F., O. Vaselli, F. Cuccoli, A. Buccianti, B. Nisi, E. Lognoli and G. Montegrossi (2009). A geochemical multi-methodological approach in hazard assessment of $\mathrm{CO}_{2}$-rich gas emissions at Mt. Amiata volcano (Tuscany, central Italy), Water Air Soil Poll. Focus, 9, 117-127; doi:10.1007/s11267-008-9198-2.

Tonani, F., and G. Miele (1991). Methods for measuring flow of carbon dioxide through soil in volcanic setting, In: International Conference on Active Volcanoes and Risk Mitigation, Napoli.
Van Gardingen, P.R., J. Grace, D.D. Harkness, F. Miglietta and A. Raschi (1995). Carbon dioxide at an Italian mineral spring. Measurement of average $\mathrm{CO}_{2}$ concentration and air temperature, Agricultural and Forest Meteorology, 73, 17-27.

Vaselli, O., F. Cuccoli, A. Buccianti, B. Nisi, E. Lognoli, L. Lombardi, S. Moretti, L. Capannesi, E. Ramaldi, F. Tassi, G. Montegrossi, B. Capaccioni and A. Minissale (2006a). Le emanazioni gassose nel Comune di Castiglione d'Orcia, Final Report of the Agreement between the Department of Earth Sciences of Florence and the Civil Protection of Tuscany, July 2006, $120 \mathrm{pp}$.

Vaselli, O., F. Tassi, G. Montegrossi, B. Capaccioni and L. Giannini (2006b). Sampling and analysis of volcanic gases, Acta Vulcanol., 18, 65-76.

Vaselli, O., F. Tassi, F. Capecchiacci, B. Nisi and D. Tardani (2011). Monitoraggio della $\mathrm{CO}_{2}$ e dei Composti Organici Volatili (COV) nella Miniera del Siele. Final Report of the Agreement between the Department of Earth Sciences of Florence and the Presidency of the Park of Mt. Amiata Mines, 35 pp.

Vaselli, O., P. Higueras, B. Nisi, J.M. Esbrì, J. Cabassi, A. Martìnez-Coronado, F. Tassi and D. Rappuoli (2013). Distribution of gaseous $\mathrm{Hg}$ in the Mercury mining district of Mt. Amiata (Central Italy): A geochemical survey prior the reclamation project, Environ. Res., 125, 179-187.

Zitellini, N., F. Trincardi, M. Marani and A. Fabbri (1986). Neogene tectonics of the Northern Tyrrhenian sea, G. Geol. Ser. 3, 48, 25-40.

${ }^{\star}$ Corresponding author: Barbara Nisi, CNR-IGG - Istituto di Geoscienze e Georisorse, Pisa, Italy; email: b.nisi@igg.cnr.it.

(C) 2014 by the Istituto Nazionale di Geofisica e Vulcanologia. All rights reserved. 